https://helda.helsinki.fi

\title{
The fate of the Antennae galaxies
}

\section{Lahén, Natalia}

2018-04

Lahén, N , Johansson , P H , Rantala , A , Naab , T \& Frigo , M 2018 , ' The fate of the Antennae galaxies ' , Monthly Notices of the Royal Astronomical Society , vol. 475 , no. 3 , pp. 3934-3958 . https://doi.org/10.1093/mnras/sty060-

http://hdl.handle.net/10138/309984

https://doi.org/10.1093/mnras/sty060-

publishedVersion

Downloaded from Helda, University of Helsinki institutional repository.

This is an electronic reprint of the original article.

This reprint may differ from the original in pagination and typographic detail.

Please cite the original version. 


\title{
The fate of the Antennae galaxies
}

\author{
Natalia Lahén, ${ }^{1 \star}$ Peter H. Johansson, ${ }^{1}$ Antti Rantala, ${ }^{1}$ Thorsten Naab ${ }^{2}$ \\ and Matteo Frigo ${ }^{2}$ \\ ${ }^{1}$ Department of Physics, University of Helsinki, Gustaf Hällströmin katu 2a, FI-00014 Helsinki, Finland \\ ${ }^{2}$ Max Planck Institute for Astrophysics, Karl-Schwarzschild-Str. 1, D-85748 Garching, Germany
}

Accepted 2018 January 5. Received 2017 December 9; in original form 2017 August 31

\begin{abstract}
We present a high-resolution smoothed particle hydrodynamic simulation of the Antennae galaxies (NGC 4038/4039) and follow the evolution 3 Gyr beyond the final coalescence. The simulation includes metallicity-dependent cooling, star formation, and both stellar feedback and chemical enrichment. The simulated best-match Antennae reproduce well both the observed morphology and the off-nuclear starburst. We also produce for the first time a simulated two-dimensional (2D) metallicity map of the Antennae and find good agreement with the observed metallicity of off-nuclear stellar clusters; however, the nuclear metallicities are overproduced by $\sim 0.5 \mathrm{dex}$. Using the radiative transfer code SKIRT, we produce multiwavelength observations of both the Antennae and the merger remnant. The 1-Gyr-old remnant is well fitted with a Sérsic profile of $n=7.07$, and with an $r$-band effective radius of $r_{\mathrm{e}}=1.6 \mathrm{kpc}$ and velocity dispersion of $\sigma_{\mathrm{e}}=180 \mathrm{~km} \mathrm{~s}^{-1}$ the remnant is located on the Fundamental Plane of early-type galaxies (ETGs). The initially blue Antennae remnant evolves on to the red sequence after $\sim 2.5 \mathrm{Gyr}$ of secular evolution. The remnant would be classified as a fast rotator, as the specific angular momentum evolves from $\lambda_{\mathrm{Re}} \approx 0.11$ to 0.14 during its evolution. The remnant shows ordered rotation and a double peaked maximum in the mean 2D line-of-sight velocity. These kinematical features are relatively common amongst local ETGs and we specifically identify three local ETGs (NGC 3226, NGC 3379, and NGC 4494) in the ATLAs ${ }^{3 \mathrm{D}}$ sample, whose photometric and kinematic properties most resemble the Antennae remnant.
\end{abstract}

Key words: methods: numerical-galaxies: evolution-galaxies: individual: NGC 4038/4039 - galaxies: kinematics and dynamics - galaxies: starburst.

\section{INTRODUCTION}

In the early days of galaxy studies, early-type galaxies (ETGs) were considered to be a homogeneous class of galaxies characterized by their nearly spherical shapes, old stellar populations, and the lack of cold gas and ongoing star formation (Faber \& Jackson 1976; Kormendy 1977; Djorgovski \& Davis 1987). However, it is now well established that the fine structural and kinematic properties of elliptical galaxies correlate with their absolute magnitudes (Davies et al. 1983; Bender, Saglia \& Gerhard 1994; Kormendy \& Bender 1996; Thomas et al. 2005). In general, brighter ellipticals $\left(M_{\mathrm{B}} \lesssim\right.$ -21.5) typically have boxy isophotes, cored central surface brightness profiles, and often show radio and X-ray emission (e.g. Faber et al. 1997; Kormendy et al. 2009; Cappellari 2016). Bright massive ellipticals are also typically slowly rotating $(v / \sigma<0.1)$ moderately triaxial systems, with often peculiar properties such as kinematic twists and kinematically decoupled components (e.g. Cappellari

^E-mail: natalia.lahen@helsinki.fi et al. 2007; Emsellem et al. 2011; Krajnović et al. 2011). Fainter ETGs $\left(-21.5 \lesssim M_{\mathrm{B}} \lesssim-18\right)$, on the other hand, are in general oblate and have typically more discy isophotes, cuspy central surface brightness profiles and typically show no significant X-ray emission (e.g. Kormendy et al. 2009; Kormendy \& Bender 2012). These galaxies are also rotating faster $(v / \sigma \sim 1)$ and are intrinsically more anisotropic systems than slowly rotating ellipticals (e.g. Cappellari et al. 2007; Emsellem et al. 2011; Cappellari 2016).

The first models attempting to describe the formation of ETGs invoked a monolithic collapse (Eggen, Lynden-Bell \& Sandage 1962), whereas more modern galaxy formation models are steeped in the hierarchical picture of structure formation, in which galaxies grow bottom-up through mergers and gas accretion (White \& Rees 1978). In recent years, a consensus has tentatively emerged in which massive, slowly rotating ETGs are believed to have assembled through a two-stage process. In this picture, the early assembly is dominated by rapid in situ star formation fuelled by cold gas flows and hierarchical merging of multiple star-bursting progenitors, whereas the later growth at lower redshifts, below $z \lesssim 2-3$ is dominated by a more quiescent phase of accretion of stars formed mainly in 
progenitors outside the main galaxy (e.g. Naab, Johansson \& Ostriker 2009; Oser et al. 2010; Feldmann, Carollo \& Mayer 2011; Johansson, Naab \& Ostriker 2012; Wellons et al. 2015; Naab \& Ostriker 2017).

On the other hand, some of the lower mass ETGs might have formed instead in the merger scenario, in which elliptical galaxies form as result of the merger of two disc galaxies (Toomre 1977; Negroponte \& White 1983; Barnes \& Hernquist 1992; Naab \& Ostriker 2017). Much of the theoretical understanding of the merger scenario has been gained using idealized isolated merger simulations, with the first studies employing purely collisionless models (e.g. White 1978; Aarseth \& Fall 1980); later studies included also the gaseous component (e.g. Hernquist \& Katz 1989; Barnes \& Hernquist 1996; Naab, Jesseit \& Burkert 2006) and increasingly sophisticated models for gas cooling, star formation, and the feedback from both supernovae and supermassive black holes (e.g. Springel, Di Matteo \& Hernquist 2005; Cox et al. 2006a; Johansson, Naab \& Burkert 2009a; Johansson, Burkert \& Naab 2009b; Teyssier, Chapon $\&$ Bournaud 2010; Hopkins et al. 2013; Hayward et al. 2014). These idealized merger simulations demonstrated that kinematically cold discs can be transformed into hot spheroidal systems with ellipticallike properties (e.g. Negroponte \& White 1983; Barnes \& Hernquist 1992). The merger mass-ratio was found to have a significant impact on the merger remnants, with more unequal-mass mergers resulting in remnants with faster rotation and more discy isophotes, whereas more equal-mass mergers would result in slower rotation and somewhat more boxy isophotes (e.g. Naab \& Burkert 2003; Bournaud, Jog \& Combes 2005; Cox et al. 2006b; Jesseit et al. 2009; Hoffman et al. 2010; Bois et al. 2011; Naab \& Ostriker 2017).

Although the merging of disc galaxies might not be the dominant formation mechanism for the majority of ETGs (e.g. Naab \& Ostriker 2009), they are nevertheless observed in the nearby Universe. The Antennae galaxies (NGC 4038/4039) are the nearest and arguably best studied example of an ongoing major merger of two gas-rich spiral galaxies. This system contains two clearly visible, still distinct discs, together with the signature feature of a beautiful pair of narrow elongated tidal tails extending to a projected size of $20^{\prime}$, which corresponds to $\sim 100 \mathrm{kpc}$ at an estimated distance of $20 \mathrm{Mpc}$. The exact distance to the Antennae system is still surprisingly uncertain. The often quoted value of $\sim 20 \mathrm{Mpc}$ has been derived from the systemic recession velocity using Hubble constant of $H_{0}=72 \mathrm{~km} \mathrm{~s}^{-1} \mathrm{Mpc}^{-1}$ with or without corrections for deviations of the Hubble flow from linearity due to various attractors (Whitmore et al. 1999; Schweizer et al. 2008). Significantly smaller distances of 13.3-13.8 Mpc have also been determined based on the photometry of the tip of the red giant branch (Saviane, Hibbard \& Rich 2004; Saviane et al. 2008). However, recent observations of the type Ia supernova 2007sr and Cepheid variables in the Antennae seem to agree on a distance of $\sim 22 \mathrm{Mpc}$ in relatively good agreement with the traditional distance (Saviane et al. 2008; Riess et al. 2011; Fiorentino, Musella \& Marconi 2013; Tully et al. 2013), although distances in excess of $\sim 25 \mathrm{Mpc}$ have also been advocated based on the brightness and decay time of the light curve of the supernova 2007sr (Nelemans et al. 2008).

The proximity of the Antennae galaxies and the availability of detailed high-quality observations covering the entire spectrum from radio to X-ray (e.g. Whitmore et al. 1999; Fabbiano, Zezas \& Murray 2001; Wang et al. 2004; Hibbard et al. 2005; Zezas et al. 2006; Bigiel et al. 2015), including recent ALMA observations (Herrera et al. 2012; Whitmore et al. 2014), makes this system an ideal laboratory for understanding the physics of merger-induced starbursts through comparison with high-resolution simulations. In galaxy mergers, the interaction typically induces strong spiral arms and extended tidal tails in the merging galaxies. The interaction also gives rise to central tidal torques that efficiently transport gas to the centres of the galaxies (Barnes \& Hernquist 1991), where the rapid build-up of dense gas typically results in central starbursts (Mihos \& Hernquist 1996). However, the Antennae system is particularly interesting, as currently the majority of the star formation activity is not observed in the nuclei, but rather in a dusty overlap region between the merging galactic discs (Mirabel et al. 1998; Wang et al. 2004; Ueda et al. 2012), making this system a rare extranuclear starburst.

The Antennae galaxies have been a favourite and natural target for numerical simulations, with the very first simulation by Toomre $\&$ Toomre (1972) already reproducing the correct trends in the morphology of the tidal arms. Refinements that included self-consistent multicomponent galaxy models consisting of a bulge, disc, and dark matter halo (Barnes 1988) and finally models that also included gas and star formation (Mihos, Bothun \& Richstone 1993) also reproduced the general morphology of the Antennae well. The introduction of adaptive-mesh refinement techniques together with stellar feedback models have enabled a more detailed modelling of the star formation histories of the Antennae galaxies (Teyssier et al. 2010; Renaud, Bournaud \& Duc 2015). However, the star formation in all of these simulations was mostly centrally concentrated, thus the observed elevated star formation rate (SFR) in the overlap region was not reproduced. On the contrary, more recent simulations in which the time of best match is placed after the second encounter, shortly before the final coalescence, are able to produce a gas-rich star-forming overlap region in the Antennae system (Karl et al. 2008, 2010, 2013; Kotarba et al. 2010; Karl, Fall \& Naab 2011). This is also the orbital configuration used in this paper.

In this paper, we study the fate of the Antennae galaxies. The main motivation of this paper is twofold. First, we want to produce an accurate simulation of the Antennae galaxies, a bona fide representation of a specific real galaxy merger. This includes a detailed dynamical model, elaborate star formation, and feedback models including metal enrichment, and the production of mock images for a direct comparison with the observations. Secondly, we evolve the simulation past the best present-day match and into the future in order to study whether the resulting Antennae merger remnant will evolve into an low-mass discy ETG, as predicted by the merger scenario. Thus, the aim is to test whether the ETG formation through galaxy mergers is a viable process in the local Universe and whether this process can explain the formation of some of the observed local low-mass ETGs. The Antennae galaxies provide us also with a prime example of a present-day major merger of two spiral galaxies with significant star formation and their evolutionary state can be linked to the more luminous LIRGs and ULIRGs (luminous/ultraluminous infrared galaxies) that are thought to be very gas-rich disc major mergers undergoing extreme star formation and morphological transition to elliptical galaxies (e.g. Genzel et al. 1998; Dasyra et al. 2006a,b; Väisänen et al. 2017).

We produce mock images of the simulated Antennae merger and the dynamically relaxed merger remnant by combining stellar population synthesis and radiative transfer modelling (Bruzual \& Charlot 2003; Groves et al. 2008; Camps \& Baes 2015). The resulting photometric images of the Antennae and the merger remnant are compared to the present-day Antennae and a sample of local ETGs, respectively. Our multiwavelength radiative transfer analysis goes significantly beyond the radiative transfer modelling study presented in Karl et al. (2013), which found a good match between their simulated Antennae and the far-infrared observations 
performed by the Herschel-PACS instrument (Klaas et al. 2010). In addition, we also construct two-dimensional (2D) kinematic maps of the merger remnant using KINEMETRY methods (Krajnović et al. 2006; Naab et al. 2014). Recently, several observational surveys, such as ATLAS $^{3 D}$ (e.g. Cappellari et al. 2011; Bois et al. 2011), CALIFA (e.g. Sánchez et al. 2012; Wild et al. 2014), and SAMI (e.g. Bryant et al. 2015), that employ integral field units have provided detailed kinematic maps of local ETGs. Here, we study the kinematics of the future Antennae remnant in order to determine whether a local look-alike exists.

In Section 2, we discuss the details of our simulations, including the setting up of the initial conditions. The necessary postprocessing tools that are required for the analysis are described in Section 3. In Section 4, we show the simulated Antennae galaxies at the time of the best match and compare the derived morphology, SFR, and spectral energy distribution (SED) to observations. We discuss the intrinsic properties, such as the shape, metallicity, and velocity structure, of the simulated merger remnant in Section 5. The photometric light profile and colour of the future Antennae remnant is described in Section 6. In Section 7, we construct kinematic maps of the Antennae remnant and derive its rotational properties. In Section 8, we search for a best match to our simulated Antennae remnant in the observational ATLAS ${ }^{3 \mathrm{D}}$ catalogue and demonstrate that the future Antennae remnant will have properties reminiscent of local ETGs. Finally, in Section 9, we summarize our results and present our conclusions.

\section{SIMULATIONS}

\subsection{Simulation code}

Our simulations are run using the $N$-body smoothed particle hydrodynamic (SPH) code GADGET-3 (Springel 2005) using specifically the SPHGal version of the code (Hu et al. 2014; Eisenreich et al. 2017). SPHGal includes several improvements of the hydrodynamics implementation, which we briefly review here. The spline kernel of standard GADGET-3 has been replaced by a Wendland $C^{4}$ kernel (Dehnen \& Aly 2012) and the number of neighbours in the SPH smoothing kernel has been increased to 100 . The code uses the pressure-entropy formulation of SPH (Hopkins 2013; Saitoh \& Makino 2013), a higher order estimate of velocity gradients (Cullen $\&$ Dehnen 2010), a modified artificial viscosity switch with a modified strong limiter (Morris \& Monaghan 1997; Cullen \& Dehnen 2010), and artificial conduction of thermal energy (Price 2008). Finally, SPHGal employs a time step limiter that activates particles receiving feedback energy and keeps the time steps of neighbouring particles within a factor of 4 of each other. Together these changes reduce the numerical artefacts present in the fluid mixing that afflicted the original GADGET-3 version, and also considerably improve the convergence rate of the SPH calculation.

\subsection{Sub-grid models}

Astrophysical processes such as metallicity-dependent cooling, star formation, stellar feedback, as well as metal production and metal diffusion are followed using sub-resolution models originally developed by Scannapieco et al. $(2005,2006)$ and later extended by Aumer et al. (2013). The gas in the simulation cools with a rate dependent on the current gas temperature, density, and metal abundances. The cooling rates are adopted from Wiersma, Schaye \& Smith (2009) (see also Aumer et al. 2013) for an optically thin gas in ionization equilibrium and the cooling rates are calculated on an element-by-element basis taking into account the effects of uniform redshift-dependent ionizing UV/X-ray background Haardt \& Madau (2001), with the background calculated at $z=0$ for our case. The cooling rates are tabulated for temperatures between $10^{2} \mathrm{~K} \leq$ $T \leq 10^{9} \mathrm{~K}$ but below $T=10^{4} \mathrm{~K}$ the cooling becomes inefficient due to the lack of molecular cooling in the current model.

Gas particles with densities $\rho_{\mathrm{g}}$ above the threshold density of $\rho_{\text {crit }}=1.6 \times 10^{-23} \mathrm{~g} \mathrm{~cm}^{-3}$ corresponding to $n_{\mathrm{H}}=10 \mathrm{~cm}^{-3}$, and with temperatures below $T<12000 \mathrm{~K}$, are eligible for star formation. The probability for a gas particle to turn into a stellar particle is given by $1-\mathrm{e}^{-p}$, where the coefficient $p$ is defined using the local dynamical time $t_{\text {dyn }}$ as

$p=\epsilon_{\mathrm{SFR}} \frac{\Delta t}{t_{\mathrm{dyn}}}=\epsilon_{\mathrm{SFR}} \Delta t \sqrt{4 \pi G \rho_{\mathrm{g}}}$,

where $\Delta t$ is the length of the current simulation time step and the star formation efficiency is set to $\epsilon_{\mathrm{SFR}}=0.02$.

Stellar particles couple to the surrounding gaseous particles through heating and mass transfer with kinetic feedback and chemical enrichment from supernovae type Ia (SNIa), type II (SNII), and asymptotic giant branch (AGB) stars. The stellar and gaseous abundances of 11 individual elements $(\mathrm{H}, \mathrm{He}, \mathrm{C}, \mathrm{Mg}, \mathrm{O}, \mathrm{Fe}, \mathrm{Si}, \mathrm{N}$, $\mathrm{Ne}, \mathrm{S}, \mathrm{Ca}$ ) evolve based on models of chemical release rates from Iwamoto et al. (1999) for SNIa, Woosley \& Weaver (1995) for SNII and Karakas (2010) for AGB stars, respectively. Variations in the metallicity between neighbouring gaseous particles are smoothed using the diffusion implementation of Aumer et al. (2013) that employs the definition for the diffusion coefficient from Shen, Wadsley \& Stinson (2010), with the diffusion coefficient pre-factor set to 0.05 . Small changes in the diffusion coefficient are not expected to qualitatively change the derived metallicity profiles.

Stellar feedback begins with SNII explosions 3 Myr after the formation of a stellar particle, followed by SNIa and AGB feedback from 50 Myr onwards. SNII feedback is released only once, whereas the SNIa and AGB feedback are released continuously from each stellar particle individually in $50 \mathrm{Myr}$ intervals for a total time period of 10 Gyr. The particle-by-particle SNIa/AGB ejecta release rates decay proportionally to $t^{-1}$ (Maoz et al. 2011), with a total release of 2 SNIa per formed $1000 \mathrm{M}_{\odot}$. Each stellar particle flagged to give $\mathrm{SN}$ feedback releases an amount of energy equalling

$E_{\mathrm{SN}}=\frac{1}{2} m_{\mathrm{eject}} v_{\mathrm{SN}}^{2}$

into the interstellar matter, where $m_{\text {eject }}$ is the mass of the SN ejecta and $v_{\mathrm{SN}}=4000 \mathrm{~km} \mathrm{~s}^{-1}$ is the $\mathrm{SN}$ ejecta velocity. The metallicitydependent ejecta mass is distributed amongst the 10 nearest gas particles weighted by the corresponding SPH smoothing kernel of the stellar particle.

The thermal and kinetic feedbacks are distributed to the gas particles within the smoothing kernel in three phases, depending on the distance of the receiving particle from the SN (see Cioffi, McKee $\&$ Bertschinger 1988 for a review of the evolutionary phases of SN remnants). The transition radii between the different phases depend on the ejecta mass and velocity, as well as on the local gas density; see Núñez et al. (2017) for a detailed description of the transition radii and the distribution of the $\mathrm{SN}$ energy. The three phases are the free expansion (FE) phase with momentum conservation, the adiabatic Sedov-Taylor (ST) phase with heating (Taylor 1950; Sedov 1959), and the snowplow (SP) phase with efficient radiative cooling (McKee \& Ostriker 1977; Blondin et al. 1998). Gas particles in the FE region, i.e. closest to the $\mathrm{SN}$, receive only kinetic energy. The transition between the FE and ST phases is set at the radius 
Table 1. Parameters for the progenitor disc galaxies.

\begin{tabular}{lcc}
\hline Property & NGC 4038 & NGC 4039 \\
\hline$M_{\text {vir }}{ }^{a}$ & 55.2 & 55.2 \\
$M_{\text {disc, stellar }}$ & 3.3 & 3.3 \\
$M_{\text {disc, gaseous }}$ & 0.8 & 0.8 \\
$M_{\text {bulge }}$ & 1.4 & 1.4 \\
$r_{\text {disc }}{ }^{b}$ & 6.28 & 4.12 \\
$z_{\text {disc }}$ & 1.26 & 0.82 \\
$r_{\text {bulge }}$ & 1.26 & 0.82 \\
$c$ & 15 & 15 \\
$\lambda$ & 0.10 & 0.07 \\
$i^{c}$ & 60 & 60 \\
$\omega$ & 30 & 60 \\
\hline
\end{tabular}

Notes. ${ }^{a}$ Masses in $10^{10} \mathrm{M}_{\odot}$.

${ }^{b}$ Scale radii and heights in kpc.

${ }^{c}$ Angles in degrees.

within which the shocked ISM mass exceeds the SN ejecta mass. Gas particles in the ST region receive 70 per cent of the injected energy as thermal and 30 per cent as kinetic energy, as the shock ejecta are slowed down by the local ISM. In the outermost region, the velocity of the $\mathrm{SN}$ ejecta decreases further, finally dispersing the shock. In the SP region, gas is allowed to cool, and the energy is distributed similarly to the ST phase, except the amount of injected energy is reduced as a function of distance from the $\mathrm{SN}$, leading to radiative loss of energy. Energy losses in the SP phase are steeper for thermal energy than the kinetic energy, thus at the outermost radii the $\mathrm{SN}$ feedback is predominantly dominated by the kinetic component (see e.g. Núñez et al. 2017).

The AGB energy and enrichment are distributed in the same fashion as the SN feedback but only in the FE phase, with a much lower wind velocity of $25 \mathrm{~km} \mathrm{~s}^{-1}$ and lower yields adopted from Karakas (2010). The masses of the putative supermassive black holes in the Antennae galaxies are currently unknown. Thus, the simulation presented in this paper does not include supermassive black holes.

\subsection{Initial conditions}

In order to study the evolution of the Antennae merger in a controlled environment, we run a high-resolution major merger of two idealized disc galaxies. The disc galaxies represent the progenitor galaxies NGC 4038 and NGC 4039 in the ongoing merger. We set up the initial conditions following the parameters of Karl et al. (2010) (see Table 1) with additional refinements concerning the age and metallicity distribution of the stellar and gaseous particles at the start of the simulation (see Sections 2.4 and 2.5).

Both of the progenitor galaxies have virial masses of $M_{\text {vir }}=5.52 \times 10^{11} \mathrm{M}_{\odot}$. Each progenitor consists of a stellar bulge, a stellar disc, and a gaseous disc embedded in a dark matter halo. Both dark matter haloes have Hernquist density profiles (Hernquist 1990) with NFW density profile equivalent concentration parameters of $c=15$ (for a connection between the Hernquist profile and the NFW profile, see Springel et al. 2005). The dimensionless spin-parameters (see e.g. Bullock et al. 2001) are set to $\lambda_{4038}=0.1$ and $\lambda_{4039}=0.07$. The disc component of each galaxy, with a total mass of $M_{\text {disc }}=4.14 \times 10^{10} \mathrm{M}_{\odot}$, receives a fraction of the angular momentum of the halo given by the disc mass fraction $m_{\mathrm{d}}=0.075$. Each disc has a gas fraction of $f_{\mathrm{g}}=0.2$, with the remainder of the disc consisting of stars. The rotationally supported stellar discs have scale lengths of $r_{\mathrm{d}, 4038}=6.28 \mathrm{kpc}$ and $r_{\mathrm{d}, 4039}=4.12 \mathrm{kpc}$ and vertical scale heights of $z_{\mathrm{d}, 4038}=1.26 \mathrm{kpc}$ and $z_{\mathrm{d}, 4039}=0.82 \mathrm{kpc}$, set as 20 per cent of the respective scale lengths. The gaseous discs have the same scale lengths as the stellar discs, and scale heights iterated through the requirement for hydrostatic equilibrium (Springel et al. 2005). The stellar bulges with bulge-to-disc mass ratios of $1 / 3$ are set up also using the Hernquist density profile with scale lengths of $r_{\text {bulge }}=0.2 r_{\text {disc }}$, resulting in a total baryon fraction of $f_{\mathrm{b}}=0.1$ for the progenitor galaxies.

Each galaxy consists of $8.28 \times 10^{6}$ particles in total, with $2.76 \times 10^{6}$ dark matter particles, $3.31 \times 10^{6}$ stellar disc particles, $1.38 \times 10^{6}$ bulge particles, and $8.3 \times 10^{5}$ gaseous particles. Baryonic matter is thus represented by macroparticles with an initial mass resolution of $m_{\mathrm{b}}=1.0 \times 10^{4} \mathrm{M}_{\odot}$, whereas the dark matter has a mass resolution of $m_{\mathrm{DM}}=1.8 \times 10^{5} \mathrm{M}_{\odot}$. The mass resolution is therefore increased by a factor of $\sim 7$ compared to the one originally used in Karl et al. (2010). We checked for convergence by running the simulation both at the original Karl et al. (2010) resolution and at an intermediate resolution, and found similar results by adjusting only the time of best match, i.e. which particular snapshot represents best the observations. The gravitational softening lengths are set at $\epsilon_{\mathrm{b}}=13 \mathrm{pc}$ for the baryonic components and $\epsilon_{\mathrm{DM}}=55 \mathrm{pc}$ for the dark matter, respectively. The simulation was run for a total of $4.3 \mathrm{Gyr}$, which corresponds to an evolution of $3 \mathrm{Gyr}$ past the final coalescence of the galaxies, which takes place at approximately $t=1.3 \mathrm{Gyr}$.

The galaxies are set on nearly parabolic orbits with an initial separation of one virial radius, $r_{\mathrm{sep}}=r_{\text {vir }}=168 \mathrm{kpc}$ and the pericentric separation set to the sum of their disc scale lengths, $r_{\mathrm{p}}=r_{\mathrm{d}, 4038}+r_{\mathrm{d}, 4038}=10.4 \mathrm{kpc}$. The galaxies are rotated with disc inclinations of $i_{4038}=60^{\circ}$ and $i_{4039}=60^{\circ}$ and arguments of pericentre of $\omega_{4038}=30^{\circ}$ and $\omega_{4039}=60^{\circ}$. This orbital configuration has been shown to reproduce a good match to the spatial distribution and line-of-sight kinematics of the baryonic matter in the observed Antennae counterparts by Karl et al. (2010).

\subsection{Initial distribution of stellar ages}

The simulation is started $1.2 \mathrm{Gyr}$ in the past corresponding to a redshift of $z \sim 0.1$. This results in the best match between the observed and simulated Antennae at $z=0$ corresponding to a 13.7Gyr-old universe. In order to capture the feedback from stars at the start of the simulation, we assign cosmic formation times and metallicities to the stellar particles present at the beginning of the simulation. The selected ages and metallicities are derived from observationally supported star formation histories and metallicity distributions separately for the bulge, and the stellar and gaseous discs.

The stellar mass in the bulges of disc galaxies in the local universe consists predominantly of old stars. To obtain the distribution of cosmic formation times of the stellar particles in the bulge, we apply an exponentially decaying SFR

$\operatorname{SFR}_{\mathrm{b}}(t)=C e^{-\left(t-t_{0}\right) / \tau}$,

where $\tau=1 \mathrm{Gyr}$ is the star formation time-scale, $t$ is the cosmic time, $t_{0}$ is the starting time of the simulation, and $C$ is a normalization factor. We set the cosmic time at which SF begins to $500 \mathrm{Myr}$ and also assume that the contribution from SF in the bulge is negligible at the start of the simulation $(z \sim 0.1)$, reminiscent of the bulges in local disc galaxies (e.g. Morselli et al. 2017). 
The formation times of the stellar particles in the disc are acquired by assuming a linearly decaying star formation history with an initial SFR at $z \sim 0.1$ similar to a local star-forming stellar disc. The initial SFR values are obtained through an iterative process where we first make an initial estimate, set up the galaxies and run them in isolation and adjust this estimate until we have a continuous SFR transitioning from the pre-set values to the actual SFR at the start of the simulation. However, the SFR at the beginning of the simulation is mainly governed by the gas content of the galaxy, and with the gas fraction of $f_{\mathrm{g}}=0.2$, we are able to produce stable star-forming discs with initial SFR values of $0.5 \mathrm{M}_{\odot} \mathrm{yr}^{-1}$ for the extended NGC 4038 and $1.5 \mathrm{M}_{\odot} \mathrm{yr}^{-1}$ for the more compact NGC 4039 .

The ages of the individual stellar particles are obtained by integrating the time-dependent SFR and requiring that all the stellar mass in both the bulge and the disc have formed before the start of the simulation. The ages of the stellar particles are then randomly sampled from the cumulative distribution functions for both the bulge and the disc components, with an assigned age scatter of $\Delta t=100 \mathrm{Myr}$.

\subsection{Initial distribution of metals}

The initial element abundances of the stellar and gaseous particles are set by assigning uniform, radially decaying metallicity profiles. The bulge, the stellar disc, and the gaseous disc are set up with equal radial gradients. We use the Milky Way as a reference, as our progenitor galaxies have stellar masses quite similar to the Milky Way, for which stellar mass estimates range between $\sim 4$ $6.4 \times 10^{10} \mathrm{M}_{\odot}$ (e.g. Klypin, Zhao \& Somerville 2002; McMillan 2011). Moreover, our progenitor galaxies lie in the intermediate to massive end of the stellar mass-gas metallicity relation (Tremonti et al. 2004), where slight deviations in stellar mass only result in small changes in metallicity.

The radial abundance profile of each metal $X$ is linear in dex-units, and is set as

$12+\log \left(\frac{X}{\mathrm{H}}\right) \equiv\left[\frac{X}{\mathrm{H}}\right]=k\left(r_{s}-r\right)+\left[\frac{X}{\mathrm{H}}\right]_{r_{s}}$,

where $k$ is the metallicity gradient, $r_{s}$ is the disc scale radius, $r$ is the distance from the galactic centre, and $[X / \mathrm{H}]_{r_{s}}$ is the normalization abundance at $r_{s}$. The observed oxygen gradient of $0.0585 \mathrm{dex} \mathrm{kpc}-1$ for the Milky Way from Zaritsky, Kennicutt \& Huchra (1994) is used as a reference, and the gradients in the two galaxies are scaled by keeping the integrated metal abundance equal to the Milky Way abundance. For our progenitor galaxies with slightly smaller stellar masses than the Milky Way, this results in mildly steeper gradients of $k_{4038}=0.0612 \mathrm{dex} \mathrm{kpc}^{-1}$ and $k_{4039}=0.0594 \mathrm{dex} \mathrm{kpc}-1$ for NGC 4038 and NGC 4039, respectively. Abundances of the nine metals at the respective scale radii are obtained from observations of the Milky Way at a radius of $3 \mathrm{kpc}$ according to KilianMontenbruck, Gehren \& Nissen (1994) and Adelman, Robinson \& Wahlgren (1993) and are given in Table 2.

To account for the increase of the mass fraction of helium from the primordial value of $\sim 24$ per cent as elements are processed during stellar evolution, we apply a metal-dependent helium mass fraction. The helium mass fraction $Y$ has been observed to increase linearly as a function of the metal mass fraction in stars, $Z=m_{\mathrm{met}} / m_{\mathrm{tot}}$, as $Y \approx 0.24+2.4 Z$ (Ribas et al. 2000; Casagrande et al. 2007), which is very close to the relation used in the stellar population synthesis library of Bruzual \& Charlot (2003) employed in Section 3. The remaining mass ( $\sim 74$ percent) in each particle is hydrogen. This yields approximately solar metal mass fractions for the galaxies
Table 2. Initial abundances of the nine individual metals at the disc scale radii (see Table 1), and the mean metal mass fractions in the stellar and gaseous components in both of the progenitor galaxies.

\begin{tabular}{llcccc}
\hline Metal & {$\left[\frac{X}{\mathrm{H}}\right]_{r_{s}}$} & Metal & {$\left[\frac{X}{\mathrm{H}}\right]_{r_{s}}$} & Metal & {$\left[\frac{X}{\mathrm{H}}\right]_{r_{s}}$} \\
\hline $\mathrm{C}$ & 8.714 & $\mathrm{Fe}$ & 7.996 & $\mathrm{Ne}$ & 8.535 \\
$\mathrm{Mg}$ & 7.726 & $\mathrm{Si}$ & 7.547 & $\mathrm{~S}$ & 7.457 \\
$\mathrm{O}$ & 8.984 & $\mathrm{~N}$ & 8.175 & $\mathrm{Ca}$ & 6.199 \\
& $\mathrm{Z}_{4038} / \mathrm{Z}_{\odot}{ }^{a}$ & & $\mathrm{Z}_{4039} / \mathrm{Z}_{\odot}$ & & \\
Stellar & 0.992 & 1.053 & & \\
Gaseous & 0.863 & & 0.968 & & \\
Note. & ${ }^{a}$ Relative to solar metallicities $\mathrm{Z}_{\odot}=0.02$.
\end{tabular}

with a slightly higher value for the more compact progenitor NGC 4039 (see Table 2).

We compare the resulting progenitor galaxies to the observed stellar mass - gas phase metallicity $\left(M_{*}-Z\right)$ relation to verify the realism of the constructed metallicity profiles. We use the mean $[\mathrm{O} / \mathrm{H}]$ in the gaseous particles to trace the metallicity in the initial conditions. The initial stellar mass of the idealized progenitor galaxies is $\sim 4.7 \times 10^{10} \mathrm{M}_{\odot}$ and the mean gaseous oxygen abundance is $[\mathrm{O} / \mathrm{H}] \approx 8.8$, placing the progenitors 0.2 dex below the canonical observed $M_{*}-Z$ relation (Tremonti et al. 2004). However, the type of available data used as a metallicity indicator and the choice of calibration may introduce variations in the obtained metallicities of up to $0.7 \mathrm{dex}$, as pointed out by e.g. Kewley \& Ellison (2008) for similar SDSS data as used in Tremonti et al. (2004). Spectral data are also often restricted to only the central regions of galaxies due to the fibre size of the instruments, which results in a systematical overestimation of galactic metallicities (Guidi, Scannapieco $\&$ Walcher 2015). Thus, given the observational uncertainties, the progenitors are in agreement with the observed $M_{*}-Z$ relations.

Finally, the cosmic formation times of the stellar particles are sorted and assigned to the particles according to the metal mass fraction so that the least metal-rich particles are given the earliest formation times. The metallicities are given a scatter of 0.2 dex motivated by the maximum measurement error in Kilian-Montenbruck et al. (1994), distributed amongst the individual elements by weighing according to the respective abundances. We set an upper limit for the metal mass fraction of 5 percent for each particle when adding the scatter to prevent unphysically large metallicities for single particles.

\section{POST-PROCESSING TOOLS}

\subsection{Dusty radiative transfer with SKIRT}

We produce mock observations with the panchromatic radiative transfer code sKIRT (Saftly, Baes \& Camps 2014; Camps \& Baes 2015). The SEDs produced by each stellar particle are interpolated within SKIRT using the metallicity, age, and mass-dependent population synthesis models of Bruzual \& Charlot (2003, hereafter BC03), assuming a Chabrier initial mass function (IMF) (Chabrier 2003). SKIRT uses the SPH data to model the distribution of dust, which requires an approximation of the dust content within the interstellar gas. The dust-to-gas ratio in galaxies has been observed to correlate with metallicity (Issa, MacLaren \& Wolfendale 1990). Thus, in order to obtain the distribution of dust mass in each simulation snapshot processed with SKIRT, we adopt a Milky Way-like dustto-metals ratio of 0.3 (Dwek 1998). We further assume that only gaseous star-forming particles or particles with temperatures below 
$T<8000 \mathrm{~K}$ are able to contain dust, i.e. if a gas particle is too diffuse and too hot we assume that the ISM conditions are such that the dust gets destroyed.

SKIRT provides multiple dust models of which we use a Milky Way-like implementation with graphite and silicate grains and polycyclic aromatic hydrocarbon (PAH) molecules with a size distribution adapted from Zubko, Dwek \& Arendt (2004). The calorimetric properties of the different grains are adapted from Draine \& $\mathrm{Li}$ (2001) and the optical properties for the grains are obtained from Bruce Draine's website ${ }^{1}$ that summarizes results from various studies (e.g. Draine \& Lee 1984; Laor \& Draine 1993; Li \& Draine 2001). The spatial properties of dust are calculated within SKIRT on an octree grid (Saftly et al. 2013).

\subsection{Star-forming regions}

When calculating the SED of the simulated Antennae during the violent phases of the merger, special care must be taken with the distribution of the star-forming regions. The young stellar component embedded in the star-forming regions contributes to the UVoptical part of the spectrum, as well as the IR spectrum through dust absorption and re-emission. The star formation scheme of GADGET-3 is not optimal for tracing the distribution of star-forming molecular clouds, since the $\sim 10^{4} \mathrm{M}_{\odot}$ stellar particles form stochastically with roughly equal masses. Observed star-forming regions, on the other hand, have a self-similar structure over several orders of magnitude in mass, from Jupiter masses to $10^{6} \mathrm{M}_{\odot}$, reflecting the fractal nature of interstellar molecular clouds (Heithausen et al. 1998; Kramer et al. 1998; Stutzki et al. 1998). For example, young stellar clusters in the Antennae have been observed to encompass masses from $10^{4} \mathrm{M}_{\odot}$ to $10^{6} \mathrm{M}_{\odot}$ with a mass function power-law index of -2 (Zhang \& Fall 1999). In modelling the spatial distribution of the youngest stellar population we follow the implementation of Camps et al. (2016) (see also Jonsson, Groves \& Cox 2010) with slight modifications by constructing an additional population of stellar particles to be given as input to SKIRT. This particle population receives distinctive SEDs calculated using the photoionization code MAPPINGS III and incorporated within SKIRT. Briefly, the SED in these particles represents the distribution of the $\mathrm{H}_{\text {II }}$ regions and the surrounding photodissociation regions (PDRs) from which the characteristic spectral signatures of star formation are obtained. For details and the description of the MAPPINGS III code, see Groves et al. (2008) and references therein.

In order to resolve the radiation field produced by the star-forming regions, the distribution of the young stellar component is obtained by grouping and redistributing nearby macroparticles into sub-particles with an observationally motivated mass distribution. First, we combine the cold or star-forming gas particles and stellar particles with ages below $10 \mathrm{Myr}$ into gaseous aggregates with masses of $M_{\text {tot }} \approx 10^{6} \mathrm{M}_{\odot}$. Given our mass resolution and SFRs, this always results in at least a few thousand particles that are eligible for resampling. Quantities such as metallicity and density are given by mass-weighted averages within each aggregate to regain the properties of the local ISM. These aggregates are then resampled into smaller mass particles with a mass distribution drawn randomly from the observed mass function of interstellar molecular clouds $N \propto M^{-0.8}$ (Heithausen et al. 1998; Kramer et al. 1998) in the mass range of $700 \mathrm{M}_{\odot} \leq M \leq 10^{6} \mathrm{M}_{\odot}$ and distributed randomly within the volume in which the original particles resided. In this

\footnotetext{
${ }^{1}$ http://www.astro.princeton.edu/ draine/dust/dust.diel.html
}

way, the emission from the stochastic particle-based star formation prescription is distributed spatially also into the gaseous regions where stars could be forming based on the local ISM conditions. As already noted by Jonsson et al. (2010), the mass resolution of the particles with MAPPINGS III SEDs only have a small effect on the integrated SED of the processed galaxies. However, we are here interested in the spatial properties of the Antennae merger, for which the redistribution of star formation is essential.

Next, the sub-particles are assigned randomly sampled ages drawn from a cumulative distribution function assuming a constant SFR for the last $10 \mathrm{Myr}$ and into the future if necessary. The assumption of constant SFR is justified as long as the time-scale for the rate of change of the SFR is longer than the assigned $10 \mathrm{Myr}$, which is valid for all snapshots considered here. The age distribution is calculated simply from the mass of the gas aggregate $M_{\text {tot }}$ and the SFR within the aggregate, given by the total SFR in the gas particles used in constructing the aggregate. The ages of the subparticles are then used to divide the sub-particles into two categories. The sub-particles that receive ages between 0 and $10 \mathrm{Myr}$ are set as a new particle type, called PDR-particles hereafter. These particles represent the young stellar population in the GADGET-3 snapshot with additional properties required by the MAPPINGS III SED. Particles which would form in the future, i.e. the mass in the aggregate not formed during the last $10 \mathrm{Myr}$ of constant star formation, are returned to the gas population of SPH particles.

The calculation of the SED of the PDR particles from the tabulated MAPPINGS III data requires the knowledge of the physical state of the molecular cloud in which the young stellar component resides. This is achieved by providing SKIRT with additional parameters for the PDR particles. The SFR in an individual PDR particle is given by the mass $M_{\mathrm{PDR}}$ and the age of the PDR particle. However, as noted by Trayford et al. (2017), the MAPPINGS III library assumes that the SFR has been constant for $10 \mathrm{Myr}$. Therefore in order to conserve mass we assume always an age of $10 \mathrm{Myr}$ when calculating the SFRs for the PDR particles. In order to further preserve the spatial star formation distribution in the snapshots, we also scale the SFR within each aggregate to the original SFR value directly derived from the simulation. The pressure $P$ of the local ISM is obtained from the local ISM density $\rho_{\text {ISM }}$ using an equation of state of the form $P$ $\propto \rho_{\text {ISM }}$ assuming full ionization. The metallicity $Z$ is given by the mass-weighted average metallicity within the combined $10^{6} \mathrm{M}_{\odot}$ gas aggregate. The compactness $C$ of the $\mathrm{H}_{\text {II }}$ region is adopted from Groves et al. (2008) and defined as a function of $P$ and $M_{\mathrm{PDR}}$ as

$$
\log C=\frac{3}{5} \log \left(\frac{M_{\mathrm{PDR}}}{\mathrm{M}_{\odot}}\right)+\frac{2}{5} \log \left(\frac{P}{k_{\mathrm{B}} \mathrm{cm}^{-3} \mathrm{~K}}\right),
$$

where $k_{\mathrm{B}}$ is the Boltzmann constant. The covering fraction of the PDR region $f_{\mathrm{PDR}}$ describes how much of the light escapes directly versus through re-emission. The value of the covering factor is set to $f_{\mathrm{PDR}}=0.1$ following Camps et al. (2016), who validated the value of $f_{\mathrm{PDR}}$ with a parameter study using similar simulation data to ours. To obtain the size of the star-forming region, i.e. the size of the smoothing length $h$ of the PDR particle, we employ the cubic spline kernel used in the smoothing of the properties of the SPH particles within SKIRT given as $M_{\text {cloud }}=(8 / \pi) \rho_{\text {ISM }} h^{3}$, where $M_{\text {cloud }}$ is the mass of the entire star-forming region including the surrounding birth cloud. We invert the spline kernel to obtain $h$ by assuming that the $\mathrm{H}_{\text {II }}$ region and the PDR are embedded in a gas cloud that is ten times as massive, $M_{\text {cloud }}=10 M_{\mathrm{PDR}}$. Finally, in order to compensate for the dust in the added gas mass, we also give SKIRT an equivalent amount of gas particles with negative mass to be reduced from the ambient ISM in which PDR particles are present. 


\subsection{Production of mock images}

SKIRT is used to produce dust attenuated flux maps at 450 logarithmically spaced wavelengths between $0.02 \leq \lambda \leq 2000 \mu \mathrm{m}$, at different epochs during the evolution of the Antennae merger. In all the analysis in this paper, we assume a distance of $30 \mathrm{Mpc}$ to the Antennae, which is at the upper range of the observed distance estimates, but provides a good match to the morphology of the observed Antennae for our chosen initial conditions (Karl et al. 2010).

We define the SDSS resolution of $0.396 \mathrm{arcsec}_{\mathrm{pix}}{ }^{-1}$ as the default resolution of our images both to be compatible with the ATLAS ${ }^{3 \mathrm{D}}$ survey and to ease the computational load of running radiative transfer modelling on high resolution GADGET-3 snapshots. For visualization purposes, we also process the inner $20 \mathrm{kpc}$ region of the best match Antennae at a Hubble Space Telescope (HST) WFPC2-equivalent resolution of $0.1 \operatorname{arcsec}$ pix $^{-1}$ and the entire merger including the tidal tails at a lower $\sim 1 \operatorname{arcsec}$ pix $^{-1}$ resolution. All the images in this paper are oriented such that the upper and lower galaxies of our best match Antennae are the observed northern NGC 4038 and the southern NGC 4039, respectively.

The data cubes obtained from SKIRT are observed through several filters in order to produce mock images. HST ACS/WFC filters F435W, F550M, and F814W are used to study the large-scale features of the best match Antennae, and Johnson-Cousins $U, B, V$, and I filters (Bessell, Castelli \& Plez 1998) are used to produce colour composite images of the central region. The resulting mock SEDs, mock-RGB composites, and derived observables are then compared in Section 4.2 to a wide range of observations of the Antennae system such as the HST observations in the Hubble Legacy Archive (Whitmore et al. 1999).

For the photometric analysis of the merger remnant, we employ the SDSS $u, g$, and $r$ filters using the standard asinh system of magnitudes $^{2}$ (Lupton, Gunn \& Szalay 1999). In order to produce mock images that are as realistic as possible, we add to the images in each respective filter an SDSS equivalent background sky component and noise (Pawlik et al., in preparation). The SKIRT images in the SDSS bands are used to calculate the colour evolution of the merger remnant in Section 6 and the produced surface brightness profiles are analysed with the fitting tool GALFIT (Peng et al. 2010) in order to extract characteristic properties directly comparable to observational surveys such as ATLAS ${ }^{3 \mathrm{D}}$.

\section{ANTENNAE AT THE TIME OF BEST MATCH}

\subsection{Stability of the progenitor galaxies}

We begin by discussing briefly the stability of the initialized progenitor galaxies during the orbital approach by analysing the behaviour of the galaxies run in isolation for $500 \mathrm{Myr}$. The total SFR, taken here to be the sum of the sub-grid SFR in the gaseous particles in the simulation, is shown in Fig. 1 for each of the progenitors during the $500 \mathrm{Myr}$ of isolated evolution. The progenitors evolve as quiescent disc galaxies and the SFRs quickly settle to values of $0.5 \mathrm{M}_{\odot} \mathrm{yr}^{-1}$ for NGC 4038 and $1.5 \mathrm{M}_{\odot} \mathrm{yr}^{-1}$ for NGC 4039 as was iteratively established in setting up the initial conditions.

The initial radial oxygen abundance of the more extended progenitor galaxy NGC 4038 is shown in Fig. 2, compared with the abundance after $500 \mathrm{Myr}$ of isolated evolution. For the NGC 4039

\footnotetext{
${ }^{2}$ The asinh-magnitudes are identical to the standard logarithmic magnitudes at high flux values (e.g. $\leq 20 \mathrm{mag}$ in the SDSS) but decline less rapidly at lower fluxes.
}

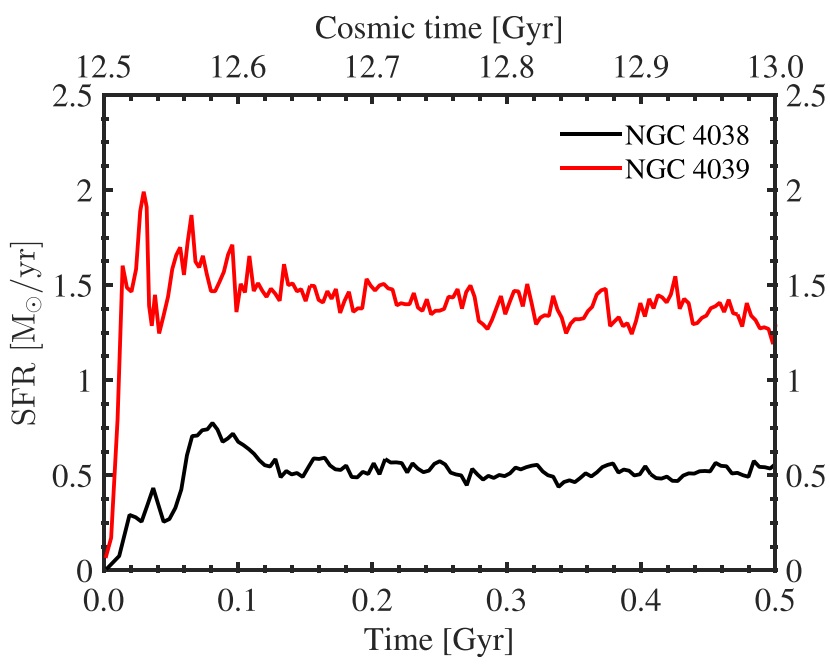

Figure 1. The SFRs of the two isolated progenitor galaxies NGC 4038 (black) and NGC 4039 (red) run separately in isolation for $500 \mathrm{Myr}$. The lower $x$-axis indicates simulation time and the upper $x$-axis gives the cosmic time.

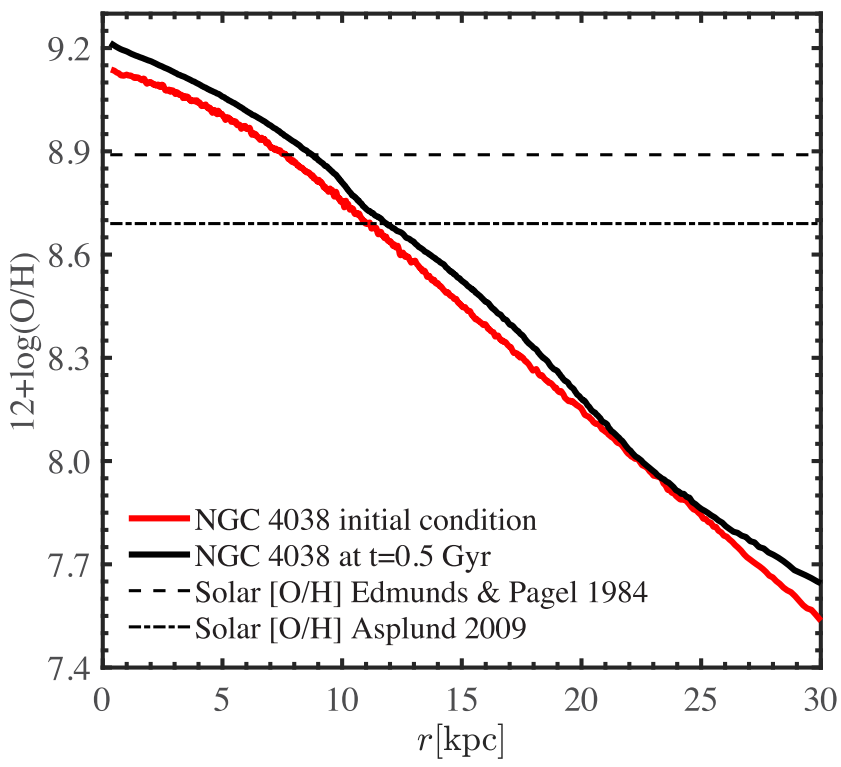

Figure 2. The radial oxygen abundance of gas in the isolated progenitor galaxy NGC 4038 run in isolation for 500 Myr. The abundance in the initial conditions is shown in red and the metallicity after $500 \mathrm{Myr}$ of evolution is shown in black. The traditional solar abundance (Edmunds \& Pagel 1984) is shown as a dashed line, together with the updated value of Asplund et al. (2009) shown as a dot-dashed line.

progenitor, the behaviour is very similar and therefore not shown separately. The radial abundance gradient is initially slightly flatter in the central region due to the maximum metal mass fraction of 5 per cent per particle defined in the initial conditions. The metallicity gradients of the gaseous and stellar discs are retained as the progenitor galaxy evolves in isolation. As a result, the gaseous oxygen abundance of NGC 4038 progenitor is enriched by less than 0.1 dex during the $500 \mathrm{Myr}$ time span.

Mock multicolour face-on and edge-on images of the NGC 4038 progenitor in the Johnson-Cousins $U+B, V$, and $I$ bands, produced using the methods described in Section 3, are shown in Fig. 3. As is evident from Figs 1-3, the progenitor discs remain stable against 

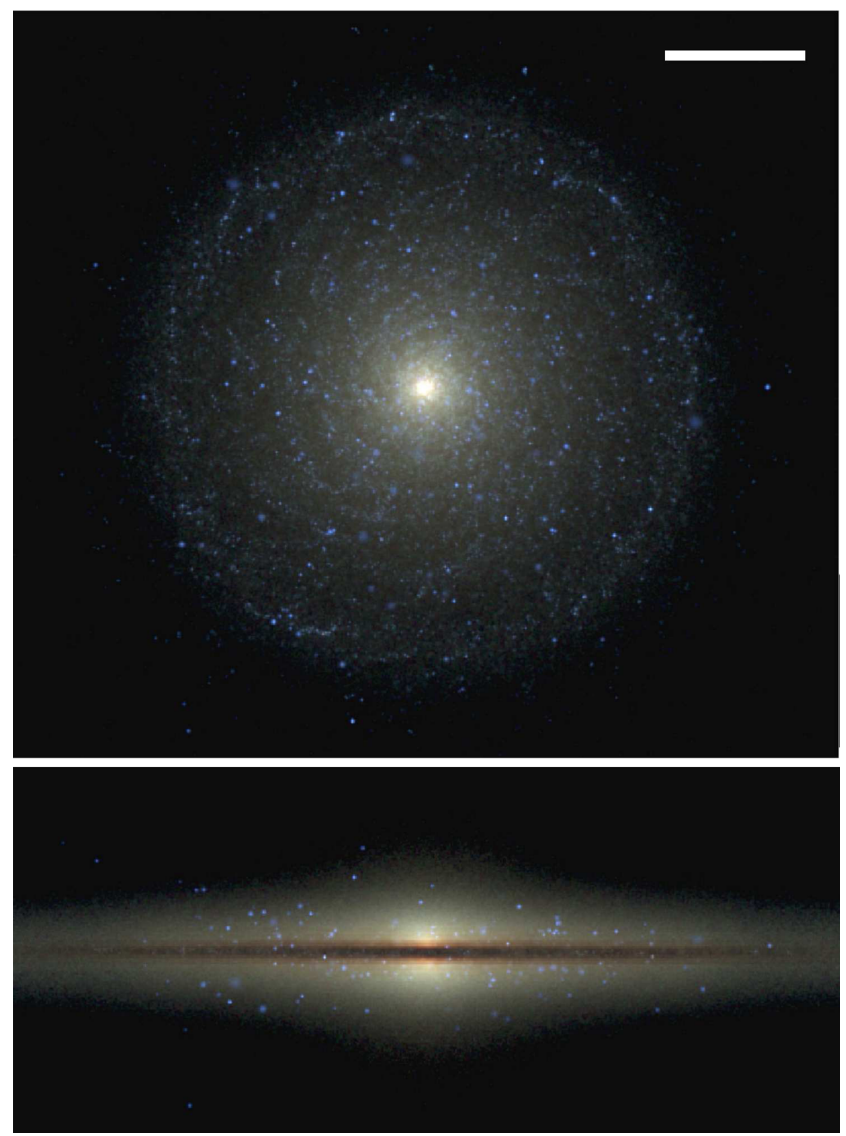

Figure 3. Mock face-on (top) and edge-on (bottom) images of the NGC4038 progenitor at a typical SDSS resolution of $0.396 \operatorname{arcsec~pix}^{-1}$ through Johnson $U+B$ (blue), $V$ (green), and $I$ (red) filters after $500 \mathrm{Myr}$ of isolated evolution. The images span $30 \mathrm{kpc}$ horizontally and the bar indicates a scale of $5 \mathrm{kpc}$. The images are produced using SKIRT (see Section 3) including the resampling of the young stellar particles.

excessive fragmentation during the $500 \mathrm{Myr}$ simulation time span. No large-scale disc instabilities are seen, which would be able to drive massive gaseous outflows or cause unpredictable amounts of star formation.

\subsection{Overall appearance}

The best match of our simulated Antennae model is produced $530 \mathrm{Myr}$ after the first and $41 \mathrm{Myr}$ after the second passages, $80 \mathrm{Myr}$ before the final coalescence of the nuclei. We show the postprocessed mock images of the best match Antennae in Fig. 4, along with two HST composite images: an image showing the large-scale features obtained from the Hubble Legacy Archive and a more detailed image showing the inner region of the merger produced by Whitmore et al. (1999). The mock images have been produced using the same ACS/WFC and Johnson filters as used in the respective observed images, as described in Section 3.3.

The most striking features of the observed Antennae, such as the tidal tails and the overlap region, are clearly visible in the mock $H S T$ images. The dust obscuration in the overlap region and the tidal arcs around the still separate nuclei follow the presence of star-forming gas. Blue light from the star-forming regions is present in similar regions and with the same clumpy structure in both the simulated and observed images. Yellow, dispersed light from the older stellar population is seen as a more extended, ambient background compared to the narrow dust lanes and blue young stellar populations.

$H S T$ observations at high resolution allow us to examine the central region in more detail and interpret the origin of the various characteristic regions using the simulation and the mock observations as references. A large number of 500- to 600-Myr-old stellar populations have been observed in the Antennae, indicating the time of the first passage (Whitmore et al. 1999). Young to intermediate age clusters with ages below $30 \mathrm{Myr}$ are found in the loop structures around NGC 4038 and in the overlap region indicating the time of the second passage (Bastian et al. 2009), and the very youngest clusters are found in the overlap region (Brandl et al. 2009). Interpreting the evolution of the simulated Antennae, we can identify the observed overlap region as the tidal bridge formed during the second passage of the galaxies. The intermediate age stellar clusters in the loop structures are less prominent tidal tail-like structures formed during the second passage, whereas the observed older population of clusters matches with our timing for the first passage and the formation time of the extended tidal tails.

\subsection{Spectral energy distribution}

The SED of the main body of the simulated Antennae is shown in Fig. 5 along with the observed values obtained from $\mathrm{NED}^{3}$ and Klaas et al. (2010). In Fig. 5, we show the stellar BC03 flux with (solid red line) and without dust attenuation (dashed red line). If dust attenuation is omitted, the simulated UV-flux would overproduce the observed values by over an order of magnitude. Including dust reprocesses, the radiation shifting a significant amount of the radiation to longer wavelengths, resulting now in turn in an underestimation of the UV-optical flux by a factor of 2-3. In Fig. 5, we also show the SED calculated both with (solid black line) and without the resampling of the young stellar component (solid red line) in order to show the effect of adding the MAPPINGS III SEDs. Direct radiation from the new population of resampled star formation regions contributes to the flux in the UV-optical part of the spectrum, increasing the flux by up to 50 per cent towards the observed values. However, even adding the MAPPINGS III SED will result in an UVoptical flux that is underestimated by a factor of $\sim 2$ with respect to the observations. Dust included around the young star-forming regions shifts again some of the emission from the young stars to infrared wavelengths, which brings additional contribution to the infrared part of the spectrum. As a result, the final infrared flux matches the observed flux in the mid-infrared at $\lambda=12 \mu \mathrm{m}$ and at long wavelengths of $\lambda>100 \mu \mathrm{m}$. However, the simulated flux is overestimated by up to a factor of 3 in the intermediate infrared range of $20<\lambda<100 \mu \mathrm{m}$. We have used a distance of $30 \mathrm{Mpc}$ following Karl et al. (2010); however, modifying this closer to the observationally favoured value of $20 \mathrm{Mpc}$ would increase the flux by a factor of $\sim 2$, increasing the optical flux close to the observed values, but would result in an even more severe overprediction of the IR flux.

We note that Karl et al. (2013) also modelled the far-infrared spectrum of their simulated Antennae galaxies using a dust model similar to ours. They were able to produce SED shapes similar to the Herschel observations by assuming a universal gas-to-dust ratio two times smaller than the canonical value of $124: 1$, yet

\footnotetext{
${ }^{3}$ The NASA/IPAC Extragalactic Database (NED) is operated by the Jet Propulsion Laboratory, California Institute of Technology, under contract with the National Aeronautics and Space Administration.
} 


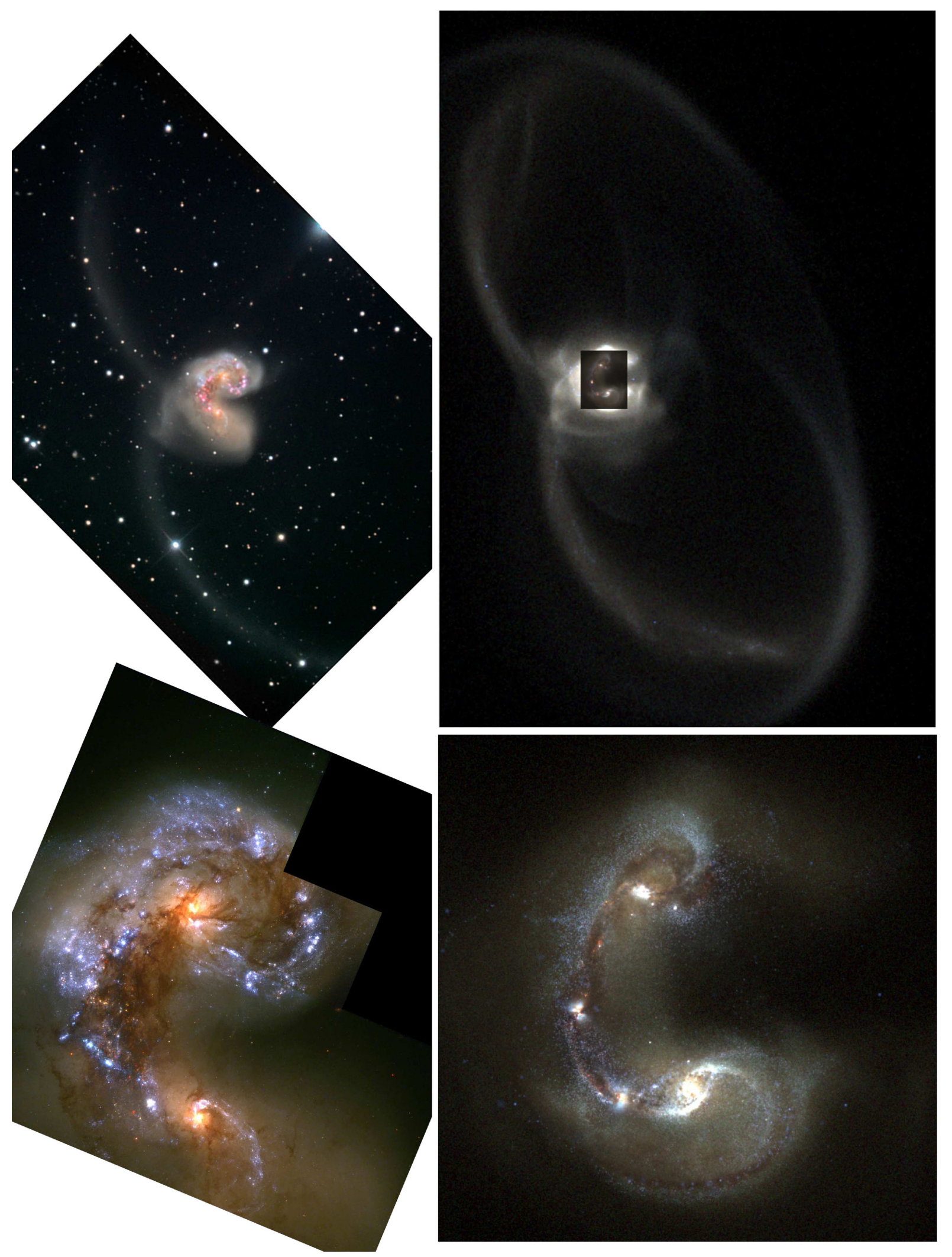

Figure 4. Left column: a colour composite from the Hubble Legacy Archive in ACS/WFC F435W (blue), F550M (green), F658N (H $\alpha+[\mathrm{N}$ II $])($ pink) and F814W (red) filters (top), and an HST composite of the inner region converted to Johnson-Cousins $U+B$ (blue), $V$ (green), and $I$ (red) bands from Whitmore et al. (1999) (bottom). Right column: simulated colour composite images of the entire Antennae system and its inner region obtained through the same respective filters (omitting the $\mathrm{H} \alpha$ band). The resolution of the mock image is $1.03 \operatorname{arcsec~pix~}^{-1}$ for the large-scale image (top) and a WFPC2 equivalent resolution of $0.1 \operatorname{arcsec} \mathrm{pix}^{-1}$ for the inner region (bottom). The tails have been highlighted using a logarithmic stretch, whereas the inlet and the zoomed image have a square root stretch. The intricate details in the simulated images are best viewed on a computer screen. 


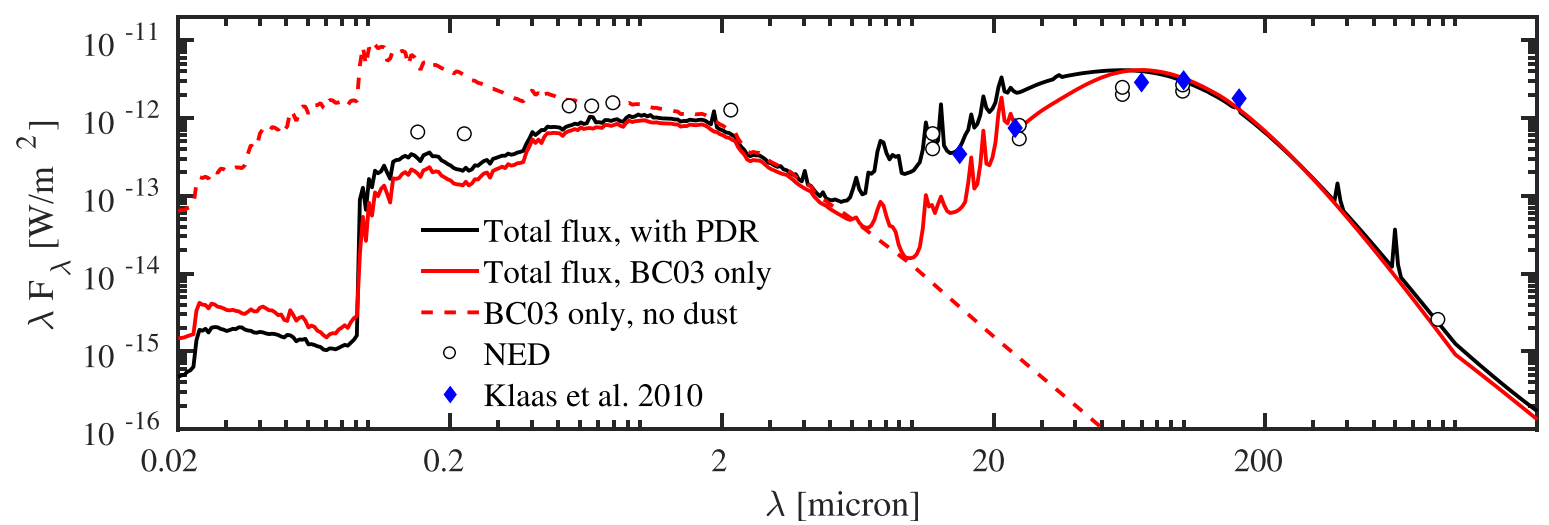

Figure 5. The mock SED of the simulated Antennae with (solid black) and without (solid red) the resampling of the young stellar component, produced with SKIRT. Both of these models include dust, whereas the dashed red curve shows the BC03 flux without dust attenuation. Observed fluxes in different wavebands obtained from NED (open markers) and Klaas et al. (2010) (blue markers) are shown for reference. The error bars in the observations are smaller than the marker size. Multiple data points at the same wavelength indicate the lower and upper limits of the observed values.

Table 3. Derived observational properties of the best match snapshot processed through sKIRT, compared to the observed values. The values are derived assuming a distance of $30 \mathrm{Mpc}$ where necessary and values in parentheses give the derived value at a distance of $20 \mathrm{Mpc}$. SFR values were calculated from the integrated IR-luminosity as described in Section 4.4, and the value for the overlap region is a lower limit from the combined SFR in the four IR-bright knots in Fig. 7.

\begin{tabular}{lllll}
\hline & Derived value & Observed value & References & Comments \\
\hline$m_{\mathrm{V}}$ & $11.1(10.2)$ & 10.37 & de Vaucouleurs \& Longo (1988) & \\
$L_{\mathrm{B}}$ & $4.16 \times 10^{10} \mathrm{~L}_{\odot}\left(1.9 \times 10^{10} \mathrm{~L}_{\odot}\right)$ & $2.9 \times 10^{10} \mathrm{~L}_{\odot}$ & de Vaucouleurs et al. (1991) & \\
$\mathrm{SFR}_{\text {total }}$ & $23.6 \mathrm{M}_{\odot} \mathrm{yr}^{-1}$ & $22.2 \mathrm{M}_{\odot} \mathrm{yr}^{-1}$ & & Regions 2-5 in Fig. 7 \\
$\mathrm{SFR}_{\text {overlap }}$ & $15.8 \mathrm{M}_{\odot} \mathrm{yr}^{-1}$ & $4.84 \mathrm{M}_{\odot} \mathrm{yr}^{-1}$ & Klaas et al. (2010) & Region 1 in Fig. 7 \\
$\mathrm{SFR}_{4038}$ & $1.0 \mathrm{M}_{\odot} \mathrm{yr}^{-1}$ & $1.17 \mathrm{M}_{\odot} \mathrm{yr}^{-1}$ & Klaas et al. (2010) & Region 6 in Fig. 7 \\
$\mathrm{SFR}_{4039}$ & $2.5 \mathrm{M}_{\odot} \mathrm{yr}^{-1}$ & $0.5 \mathrm{M}_{\odot} \mathrm{yr}^{-1}$ & Klaas et al. (2010) & Region 7 in Fig. 7 \\
$\mathrm{SFR}_{\text {arm }}$ & $0.55 \mathrm{M}_{\odot} \mathrm{yr}^{-1}$ & $0.5 \mathrm{M}_{\odot} \mathrm{yr}^{-1}$ & Klaas et al. (2010) & \\
SFR $_{\text {overlap } / \mathrm{SFR}_{\text {nuclei }}}$ & 4.5 & 2.9 & Klaas et al. (2010) & \\
\hline
\end{tabular}

still underproduced the level of far-infrared emission by at least $\sim 20-30$ per cent. Our assumption for a dust-to-metal ratio of 0.3 yields values ranging from 68:1 to $472: 1$ for our gas-to-dust ratio within the central $30 \mathrm{kpc}$. This is in a similar range as used in previous studies, but here rather than setting a uniform gas-to-dust ratio for all gaseous particles, we have a distribution of values based on the evolved metallicity of the simulation particles.

The B-band luminosity of the post-processed galaxy is $L_{\mathrm{B}}=4.16 \times 10^{10} \mathrm{~L} \odot$. Given the large uncertainty in the distance estimate, this is consistent with the observed value of $L_{\mathrm{B}}=2.9 \times 10^{10} \mathrm{~L}_{\odot}$ (Hibbard et al. 2001) measured assuming a distance of $19.2 \mathrm{Mpc}$. The derived $V$-band magnitudes of the simulated Antennae at 20 and $30 \mathrm{Mpc}$ distance are 10.2 and $11.1 \mathrm{mag}$, respectively. This is consistent with the observed $V$-band magnitude of 10.37 (de Vaucouleurs \& Longo 1988). All the observables derived from the simulations have been summarized in Table 3 along with the corresponding observed values.

\subsection{Star formation rate}

The total SFR during the simulation is shown in Fig. 6, taken again as the sum of the gaseous sub-grid SFR in the simulation and smoothed over 500 time steps that corresponds to approximately 0.2-3 Myr. The vertical bars in Fig. 6 indicate the first passage $\sim 530 \mathrm{Myr}$ ago, the present epoch at $\sim 13.7 \mathrm{Gyr}$, and when the remnant reaches an age of $1 \mathrm{Gyr}$, respectively, assuming a Hubble

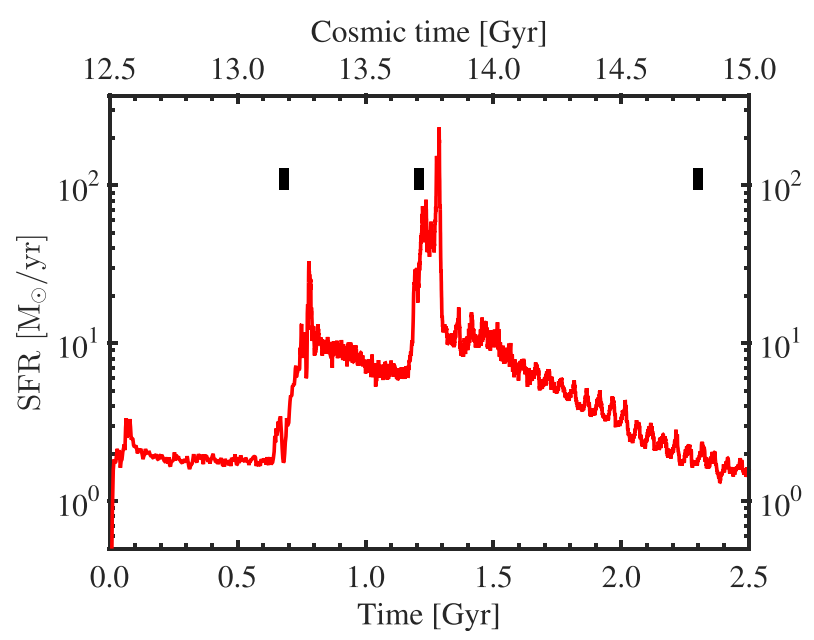

Figure 6. The SFR during the first $2.5 \mathrm{Gyr}$ of the simulation, taken as the sum of the sub-grid SFRs in the gaseous particles, averaged over 500 time steps. The lower $x$-axis indicates simulation time and the upper $x$-axis gives the cosmic time. The vertical bars indicate the time of the first pericentric passage, the time of the best match to observations $(\sim 13.7 \mathrm{Gyr})$ and the remnant age of $1 \mathrm{Gyr}$ used for the majority of the analysis related to the merger remnant. 


$$
\log \left(\Sigma_{\mathrm{SFR}}\right)\left[\log \left(\mathrm{M}_{\odot} / \mathrm{kpc}^{2} / \mathrm{yr}\right)\right]
$$

$\begin{array}{lllllllllllll}-3.5 & -3 & -2.5 & -2 & -1.5 & -1 & -0.5 & 0 & 0.5 & 1 & 1.5 & 2\end{array}$

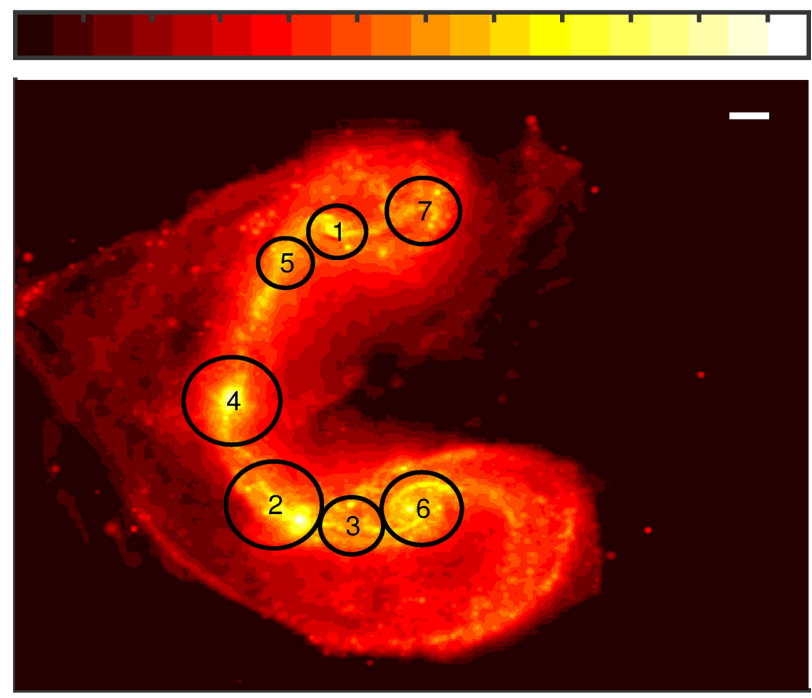

Figure 7. The spatial SFR density of the best match Antennae, as integrated on pixel-by-pixel basis from the IR luminosity (see Section 4.4). The circles encompass the IR-bright knots in the galactic nuclei (1 and 6), the overlap region (2-5), and the northern stellar arc (7) and they indicate the apertures within which the SFRs are integrated. The image spans $20 \mathrm{kpc}$, the white bar in the top-right corner indicates a scale of $1 \mathrm{kpc}$, and the image resolution is the HST ACS/WFPC2 equivalent of $0.1 \operatorname{arcsec}_{\text {pix }}{ }^{-1}$.

constant of $H_{0}=71 \mathrm{~km} \mathrm{~s}^{-1} \mathrm{Mpc}^{-1}$. The peak SFR during and immediately after the coalescence some $80 \mathrm{Myr}$ in the future reaches up to $\sim 200 \mathrm{M}_{\odot} \mathrm{yr}^{-1}$. During the following few Myr after the coalescence, the total SFR declines gradually and decreases to a value of a few solar masses per year after most of the gas ejected during the encounter falls back to the remnant. Some star formation is present in the remnant even at later age, situated mainly within the inner 1-2 kpc in a nuclear disc that forms after the final coalescence.

Following Karl et al. (2010, 2013), we also analyse the spatial distribution of star formation in the central region of the simulated merger. Observations have shown a peculiar feature, where the lower limit SFR (sum over the brightest IR knots) in the overlap region exceeds the SFR of both of the nuclei (Brandl et al. 2009; Klaas et al. 2010). This feature is also reproduced in our simulation, as can be seen in Fig. 7, where the spatial SFR density of the best match Antennae is shown. To make a connection to the observations, the SFR density in Fig. 7 has been calculated from the IR-luminosity integrated from the SKIRT SED on a pixel-by-pixel basis using the relation

$\mathrm{SFR}=1.15 \times 10^{-10}\left(\frac{L_{\mathrm{IR}}}{\mathrm{L}_{\odot}}\right) \mathrm{M}_{\odot} \mathrm{yr}^{-1}$,

where $L_{\mathrm{IR}}$ is the luminosity in the wavelength range of 8-1000 $\mu \mathrm{m}$. The definition in equation (6) is the traditional Kennicutt-relation from Kennicutt (1998) divided by 1.5 to accommodate for the use of the Chabrier IMF (Salim et al. 2007; Schiminovich et al. 2007). Following Klaas et al. (2010), we have chosen 7 IR-bright regions (indicated with numbers 1-7 in Fig. 7) with circular apertures of areas similar to the ones specified in table 3 of Klaas et al. (2010). The integrated SFRs are summarized in Table 3.

The simulated SFRs in the nuclei (regions 1 and 6) are $\mathrm{SFR}_{4038}$ $\approx 1.0 \mathrm{M}_{\odot} \mathrm{yr}^{-1}$ and $\mathrm{SFR}_{4039} \approx 2.5 \mathrm{M}_{\odot} \mathrm{yr}^{-1}$, whereas the total SFR in the IR-knots in the overlap region (regions 2-5) is $\mathrm{SFR}_{\text {overlap }}$ $\approx 15.8 \mathrm{M}_{\odot} \mathrm{yr}^{-1}$. The corresponding observed values in Klaas et al. (2010) are $\mathrm{SFR}_{4038} \approx 1.17 \mathrm{M}_{\odot} \mathrm{yr}^{-1}, \mathrm{SFR}_{4039} \approx 0.5 \mathrm{M}_{\odot} \mathrm{yr}^{-1}$, and $\mathrm{SFR}_{\text {overlap }} \approx 4.84 \mathrm{M}_{\odot} \mathrm{yr}^{-1}$. On the other hand, Brandl et al. (2009) derive observed values for the SFR that are approximately 50 per cent lower than the SFR values of Klaas et al. (2010) in the nuclei of the galaxies while they obtain for the total overlap region an SFR of $5.4 \mathrm{M}_{\odot} \mathrm{yr}^{-1}$. The SFR in the north-eastern region of NGC 4038 (region 7) is measured to be $0.55 \mathrm{M}_{\odot} \mathrm{yr}^{-1}$ in our simulation and it is comparable to the observed value of $0.5 \mathrm{M} \odot \mathrm{yr}^{-1}$. The study by Karl et al. (2013) also derived SFRs in similar regions of their simulated Antennae but only by directly taking the sum of the sub-grid SFRs of the simulated gas particles. Varying the efficiency of their stellar feedback model, Karl et al. (2013) found simulated SFR values of $10.88 \mathrm{M}_{\odot} \mathrm{yr}^{-1} \leq \mathrm{SFR}_{\text {overlap }} \leq 26.09 \mathrm{M}_{\odot} \mathrm{yr}^{-1}$ for the overlap region, and $0.49 \mathrm{M}_{\odot} \mathrm{yr}^{-1} \leq \mathrm{SFR}_{4038} \leq 0.92 \mathrm{M}_{\odot} \mathrm{yr}^{-1}$, $0.44 \mathrm{M}_{\odot} \mathrm{yr}^{-1} \leq \mathrm{SFR}_{4039} \leq 2.02 \mathrm{M}_{\odot} \mathrm{yr}^{-1}$ for the nuclear regions of NGC 4038 and NGC 4039, respectively.

Some of the regions in our simulation show therefore star formation on similar scale to the observations, whereas e.g. the starforming knots in the simulated overlap region overestimate the SFR compared to observations. The total SFR of the entire Antennae has been observed to span values between 5 and $22.2 \mathrm{M}_{\odot} \mathrm{yr}^{-1}$ (Stanford et al. 1990; Zhang, Fall \& Whitmore 2001; Brandl et al. 2009; Klaas et al. 2010). The lowest values of SFR are measured as the sum of the SFR in the observed IR knots, while e.g. Klaas et al. (2010) observe an SFR twice as high outside the few brightest IR regions. Our estimate of the total SFR is $\sim 23.6 \mathrm{M}_{\odot} \mathrm{yr}^{-1}$, which is only somewhat above the upper limit of observed values. The star formation is however concentrated in rather tight knots within the brightest regions, whereas in the observed Antennae the star formation appears to be somewhat more diffuse.

The most important feature observed in the Antennae is the overlap starburst where the observed lower limit value for the ratio of star formation in the overlap region relative to the nuclei is $\left(\mathrm{SFR}_{\text {overlap }} / \mathrm{SFR}_{\text {nuclei }}\right)_{\text {obs }}=2.9-5.4$ (Brandl et al. 2009; Klaas et al. 2010). This is also reproduced in our simulation for which we find $\left(\mathrm{SFR}_{\text {overlap }} / \mathrm{SFR}_{\text {nuclei }}\right)_{\text {sim }} \approx 4.5$. In comparison, Karl et al. (2013) obtained $9.1 \leq\left(\mathrm{SFR}_{\text {overlap }} / \mathrm{SFR}_{\text {nuclei }}\right)_{\text {obs }} \leq 11.1$ for the ratio of SFR between the overlap region and the nuclei, which is larger than the observed ratio by a factor of $\gtrsim 2-3$.

\subsection{Metallicity}

High-resolution spectroscopy of stellar clusters within the Antennae galaxies has enabled studies of the spatial metallicity distribution in the ongoing merger. In general, metallicities ranging from slightly sub-solar to super-solar $\left(0.9<Z / Z_{\odot}<1.3\right)$ have been found for young- to intermediate-age $\left(<6 \times 10^{8} \mathrm{yr}\right)$ stellar clusters with typical uncertainties of 0.1-0.2 dex (Bastian et al. 2009; Lardo et al. 2015). As noted by Kewley \& Ellison (2008) and verified for the stellar clusters in the Antennae by Lardo et al. (2015), additional variations arise from the specific metallicity-indicator and calibration methods used, which may result in offsets up to 0.7 dex for the measured metallicities.

Given the uncertainties in the observed values, we calculate here the metallicity based only on the simulated properties of the particles and leave a more detailed examination using more observationally motivated metallicity proxies for a future study. The mass-weighted spatial metallicity of stellar particles within the central $10 \mathrm{kpc}$ of the simulated Antennae is shown in Fig. 8 in solar units (assuming $\mathrm{Z}_{\odot}=0.02$ ). The image is smoothed to a resolution of $100 \mathrm{pc}$, 

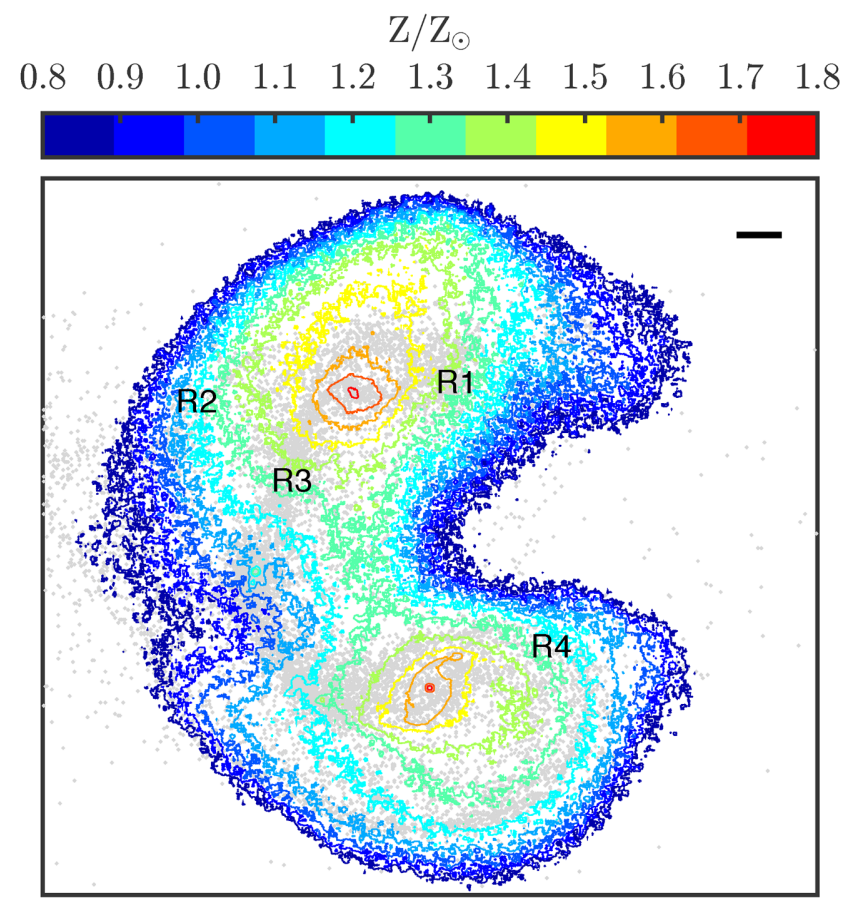

Figure 8. The contours of the pixel-by-pixel mass-weighted mean stellar metallicity of the best match Antennae in solar units for $Z_{\odot}=0.02$. Underlaid grey points show the positions of every 5 th randomly chosen gas particle. Labels R1-R4 show the approximate positions of the characteristic regions where stellar clusters have been observed (see the text for details). The image spans $20 \mathrm{kpc}$ and the black bar in the top-right corner indicates a scale of $1 \mathrm{kpc}$.

corresponding to the spatial resolution in the Bastian et al. (2009) study. Positions of every 5th randomly selected gas particle are underlaid in Fig. 8 to aide the comparison of the matter distribution of our best match Antennae and the observed regions.

Most of the structures within the central $10 \mathrm{kpc}$ show supersolar metallicities, with the nuclear regions reaching metallicities nearly twice the solar value. In addition to the nuclear regions, four regions where stellar clusters have been observed have been identified in our best match image, as indicated in Fig. 8 with labels R1-R4. Region 1 represents the arc around the nucleus of NGC 4038, region 2 represents the radial region outside the brightest optical emission at a distance of 4-5 kpc from the NGC $4038 \mathrm{nu}-$ cleus, region 3 is the northern part of the overlap region at $3 \mathrm{kpc}$ distance from the NGC 4038 nucleus, and region 4 represents the arc around the nucleus of NGC 4039. The observed metallicity ranges with maximum errors for these regions are $Z_{4038 \text {, obs }}=1.3 \pm$ $0.2 \mathrm{Z}_{\odot}, Z_{4039, \mathrm{obs}}=1.1 \pm 0.2 \mathrm{Z}_{\odot}, Z_{\mathrm{R} 1 \text {, obs }}=1.2-1.3 \pm 0.2 \mathrm{Z}_{\odot}(1.0$ $\left.1.3_{-0.23}^{+0.19} \mathrm{Z}_{\odot}\right), Z_{\mathrm{R} 2 \text {, obs }}=1.0-1.1 \pm 0.4 \mathrm{Z}_{\odot}, Z_{\mathrm{R} 3 \text {, obs }}=1.1-1.3 \pm 0.2$ $\mathrm{Z}_{\odot}\left(1.2_{-0.25}^{+0.21} \mathrm{Z}_{\odot}\right)$, and $Z_{\mathrm{R} 4 \text {, obs }}=0.9-1.1 \pm 0.1 \mathrm{Z}_{\odot}$, respectively, from Bastian et al. (2009) and in brackets from Lardo et al. (2015).

The range of values for the resulting simulated metallicities within the nuclei and regions R1-R4 are studied here within $1 \mathrm{kpc}$ circular apertures. The nuclei, where the star formation is strongly concentrated, show enhanced metallicities with $Z_{4038 \text {, sim }}=1.5-$ $1.8 \mathrm{Z}_{\odot}$ and $Z_{4039, \text { sim }}=1.4-1.8 \mathrm{Z}_{\odot}$. The derived range in the arc region at $\mathrm{R} 1$ is $Z_{\mathrm{R} 1 \text {, sim }}=1.1-1.5 \mathrm{Z}_{\odot}$. The outer regions at $\mathrm{R} 2$ and likewise at the opposite side of the NGC 4038 nucleus have a metallicity in the range $Z_{\mathrm{R} 2 \text {, sim }}=0.8-1.4 \mathrm{Z}_{\odot}$. The overlap region includes a wide range of values from 0.9 to $1.5 \mathrm{Z}_{\odot}$, with $\mathrm{R} 3$ at $Z_{\mathrm{R} 3 \text {, sim }}=$
1.2-1.5 $\mathrm{Z}_{\odot}$. The arc around the nuclear region of NGC 4039 is somewhat larger than the corresponding observed area (see Fig. 4), but the region around $\mathrm{R} 4$ and along the gaseous arc shows metallicities in the range of $Z_{\mathrm{R} 4 \text {, sim }}=1.0-1.4 \mathrm{Z}_{\odot}$. The off-nuclear regions result therefore in metallicities in broad agreement with the observed range, while the nuclear metallicities are enhanced by $0.5-0.7 \mathrm{Z}_{\odot}$ compared to the observed nuclei. A more observationally consistent method would require detailed spectral mock data and a stellar cluster selection based on age, as the observed super stellar clusters are limited to ages of $t<6.3 \times 10^{8} \mathrm{yr}$. Most importantly, the agreement on the large spatial scale adds to the credibility of our initial conditions. We thus conclude that the assumption of Milky Way-like progenitor metallicity acts as a reasonable starting point for further studies.

\section{INTRINSIC STRUCTURE OF THE MERGER REMNANT}

\subsection{Intrinsic shape and the density profile}

The merger remnant is predominantly studied $\sim 1$ Gyr after the final coalescence of the nuclei at a simulation time of $t=2.3 \mathrm{Gyr}$. During the merger, the stellar mass has grown by $1.02 \times 10^{10} \mathrm{M}_{\odot}$, corresponding to an $\sim 11$ percent increase over the initial combined stellar mass, whereas only $4.05 \times 10^{9} \mathrm{M}_{\odot}$ of the gas mass, corresponding to $\sim 25$ percent of the initial value remains in a gaseous phase. The central $30 \mathrm{kpc}$ region of the remnant consists of $1.42 \times 10^{9} \mathrm{M}_{\odot}$ gas and $8.82 \times 10^{10} \mathrm{M}_{\odot}$ stellar mass, which amounts to $\sim 35$ per cent and $\sim 85$ per cent of the total gaseous and stellar mass in the simulation volume. The remainder of the baryonic mass resides in the tidal tails and the gaseous rings around the merger remnant.

We start by studying the intrinsic three-dimensional (3D) structure of the merger remnant. A discussion of the projected apparent shape, taking into account observable effects such as dust attenuation, is deferred to Section 6. Here, we use the unweighted ellipsoidal shell method, i.e. the S1 method of Zemp et al. (2011) to define the intrinsic shape of the stellar component of the remnant. In this method, the axial ratios $b / a$ and $c / a$ are calculated iteratively in thin ellipsoidal shells to obtain the local shape as a function of radius. The calculation reveals a relatively spherical central structure with axis ratios $b / a$ and $c / a$ predominantly in the range of $0.8-0.9$ within the inner $10 \mathrm{kpc}$. The axis ratio profiles peak within $\sim 0.5 \mathrm{kpc}$ with maximum values of $b / a=0.93$ and $c / a=0.91$ and decrease gradually to values of $b / a=0.88$ and $c / a=0.8$ towards the outer radii, thus showing a spherical inner structure that transitions into a slightly more elongated shape in the outer parts.

We show in Fig. 9 the radial density profile of the matter components for the remnant $1 \mathrm{Gyr}$ after the coalescence. The combined density of all matter is dominated in the central region of the remnant by luminous matter, where most of the contribution comes from stars. Young stellar particles, formed after the first pericentric passage at approximately $t \sim 0.7 \mathrm{Gyr}$, contribute the majority of the stellar density in the inner $220 \mathrm{pc}$. These stars have either formed out of the disturbed gaseous disc during the interaction of the galaxies, or formed during the funnelling of gas into the central region. Older stars dominate the stellar density for most of the radial extent of the remnant galaxy.

The gaseous density is approximately two orders of magnitude lower than the total mass density for the entire extent of the galaxy with a profile similar to the stellar density profile. The dark matter density has a shallower profile compared to the luminous 


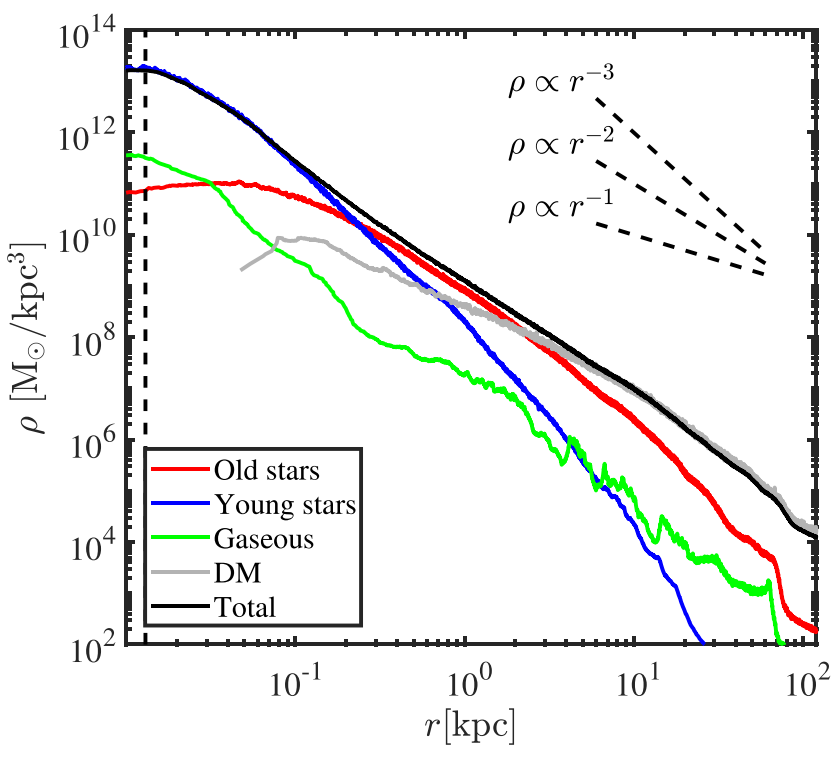

Figure 9. The density profiles of old stars (present before the first pericentric passage), young stars, gas, dark matter, and all matter combined for the 1-Gyr-old remnant. The profile of dark matter extends only down to $r \sim 50 \mathrm{pc}$ because of low number of particles in the inner region. For reference three power-law density profiles with $r^{-1}, r^{-2}$, and $r^{-3}$ are also shown. The black dashed line shows the baryonic gravitational softening of $13 \mathrm{pc}$.

components, and dark matter begins to dominate the total density from $2.5 \mathrm{kpc}$ outwards. The dark matter profile steepens beyond $12 \mathrm{kpc}$, which reflects the distribution of dark matter in the initial conditions where the scale lengths of the Hernquist halo profiles were $15 \mathrm{kpc}$.

For reference, Fig. 9 shows three power-law slopes $\rho \propto r^{-\gamma}$ with power-law indices 1, 2 and 3, where the $\gamma=2$ corresponds to an isothermal profile. The radial profile of the total density in the merger remnant is best fit with a power-law index $\gamma=2.35$. The radial lower limit of the fit was set at 2.8 times the gravitational softening at $r=36.4 \mathrm{pc}$, corresponding the limit of the force being exactly Newtonian, and the upper limit to the virial radius $r_{\text {vir }}$ $\approx 188 \mathrm{kpc}$, where the mean density reaches 200 times the critical density. Earlier studies of binary mergers and the cosmological evolution of elliptical galaxies have shown similar results for the total density profile. For example Remus et al. $(2013,2017)$ report a distribution of $\gamma$ values peaking at $\gamma \approx 2.0-2.1$ tending towards slightly lower values for lower mass galaxies. Remus et al. (2017) also note that ignoring supermassive black holes and the associated feedback in the simulation models leads to steeper values of $\gamma$ for the total density. Observations of ETGs such as the SLACS gravitational lensing study (Bolton et al. 2006, 2008) report also nearly isothermal profiles with $\gamma=2.01-2.085$ for the total density of ETGs (Koopmans et al. 2006, 2009) out to tens of effective radii (Gavazzi et al. 2007). The density slopes of both observed and simulated individual galaxies have typically a $1 \sigma$ intrinsic scatter of the order of $\pm 0.1-0.2$, while the range of $\gamma$-values for individual ETGs varies as much as \pm 0.5 . The fitted power-law index is therefore in good agreement with the upper envelope of observed $\gamma$-values. We thus conclude that the simulated Antennae merger results in a fairly representative ETG and we would most likely obtain a slightly shallower density power-law index by including also central supermassive black holes in our simulations.

\subsection{Oxygen abundance}

The intrinsic radial oxygen abundances in the 1-Gyr-old remnant are shown in Fig. 10 for the gaseous particles and the stellar particles separately, and are compared with the initial abundance of the NGC 4038 progenitor. Again we only consider the intrinsic metallicity values of the particles as given directly by the simulation. To separate the contribution from diffusion and stellar enrichment versus passive mixing due to dynamical evolution, we also show the $[\mathrm{O} / \mathrm{H}]$ profile that would result from a purely passive redistribution of the particles. This is done by tracing the initial abundances of the particles in the remnant back to the initial conditions and calculating the radial abundance as if it would have evolved passively.

For gas, both the enriched and the passively evolving $[\mathrm{O} / \mathrm{H}]$ profiles in Fig. 10 show that the initial metallicity gradients of the progenitor discs get mostly erased when the particles in the gaseous discs get redistributed during the merger.

Merging systems are typically outliers in observed metallicity relations. The metallicity profiles of interacting galaxies have been previously studied extensively using both simulations (e.g. Perez et al. 2006; Montuori et al. 2010; Rupke, Kewley \& Barnes 2010) and also observationally (e.g. Ellison et al. 2008; Rupke, Veilleux \& Baker 2008; Kewley et al. 2010; Sánchez et al. 2014), where the increasing capabilities of extensive integral field spectroscopic surveys have been particularly important. The general consensus is that the metallicity gradient flattens, as the metal-poor gas in the outer regions of the progenitors gets mixed up with the more metalrich gas in the inner regions of the interacting galaxies. The majority of this dilution is due to purely dynamical effects as already noted in Rupke et al. (2010), who did not include active star formation in their study. In agreement with previous studies, the simulated Antennae remnant in both the passively traced and the full enrichment model has an $[\mathrm{O} / \mathrm{H}]$ value below the initial metallicity of the progenitor galaxy for most of observationally accessible inner region of the galaxy.

As already pointed out in studies involving larger simulation samples, the enrichment through star formation and stellar feedback has a significant role in determining the spatial distribution of metals in both the gaseous and stellar components (e.g. Montuori et al. 2010; Perez, Michel-Dansac \& Tissera 2011; Torrey et al. 2012). Our merger simulation employing active metal enrichment results in an increase of $[\mathrm{O} / \mathrm{H}]$ by 0.2 dex over the entire radial range compared to the passively evolving simulation. We note that similar results have also been reported in Torrey et al. (2012), where they found a mean nuclear $[\mathrm{O} / \mathrm{H}]$ enhancement due to enrichment already during the pre-coalescence phase by at least 0.2 dex. The passive mixing of the gaseous gradients dominates the redistribution in the outer parts, where the abundance profile remains flat. However, when active enrichment is considered the metallicity in the innermost region of the remnant is drastically different from the purely dynamically evolving $[\mathrm{O} / \mathrm{H}]$. First, the radius outside of which the metallicity switches from underabundant to enhanced, with respect to the initial conditions, shifts $5 \mathrm{kpc}$ inwards due to the overall 0.2 dex radial enrichment. Secondly, within the innermost $8 \mathrm{kpc}$ the oxygen abundance shows a steepening gradient towards the centre of the remnant. The abundance actually exceeds the initial abundance within the innermost $1.5 \mathrm{kpc}$. This increase in metallicity is due to the central starburst resulting in enhanced star formation and enrichment.

According to the passively distributed stellar metallicity in the right-hand panel of Fig. 10, the inner $1.5 \mathrm{kpc}$ would have an inwards decreasing abundance, whereas the result from the full feedback 

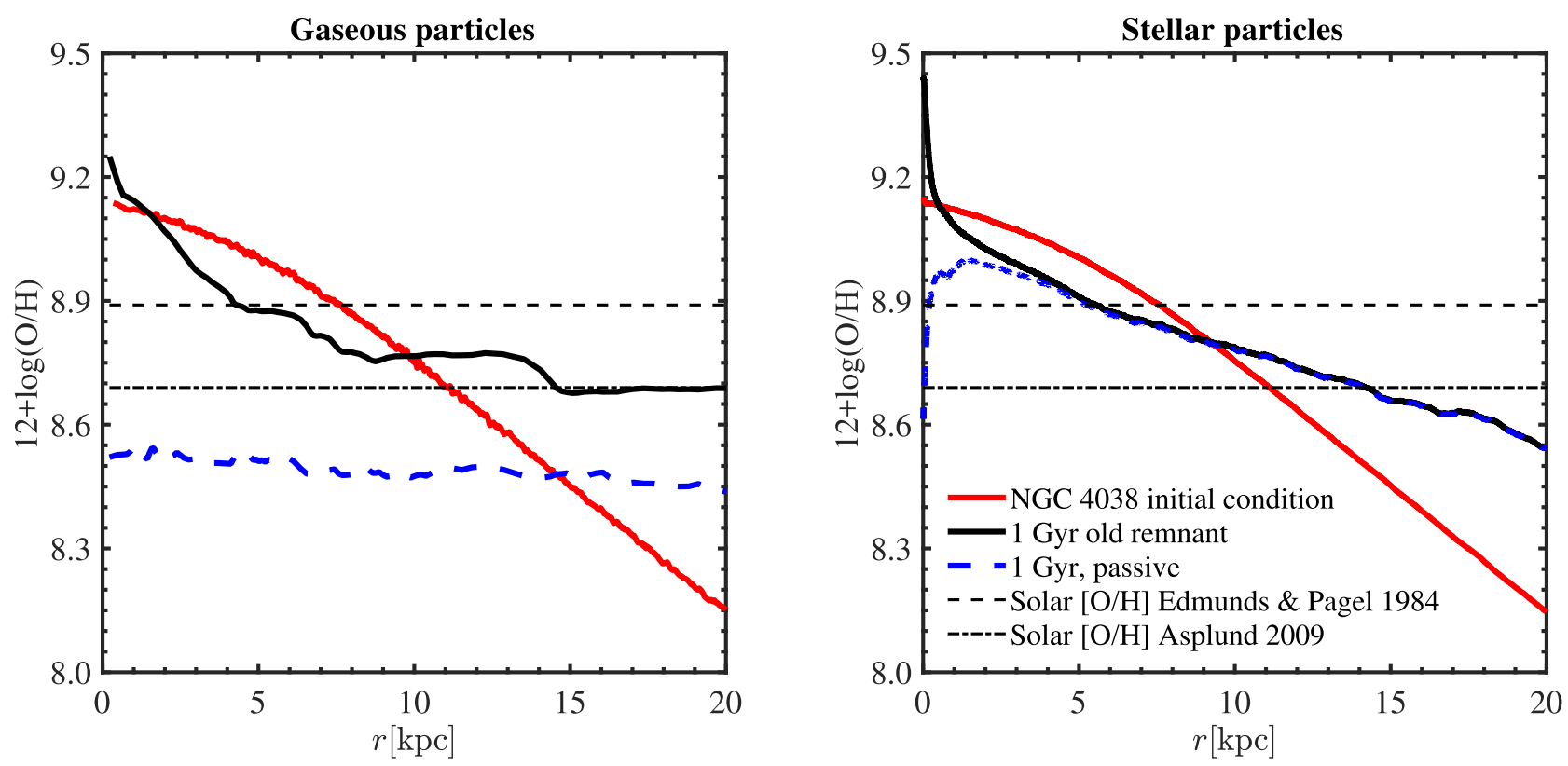

Figure 10. Gaseous (left) and stellar (right) oxygen abundance of the 1-Gyr-old remnant (black) compared to the passively evolved metallicity obtained from the initial conditions (dashed blue, see the text for details) and the initial abundance of the NGC 4038 progenitor (red). The observed solar abundances are also shown for reference.

implementation results in a super-solar enhancement of over 0.5 dex. The difference can be explained with the properties of the central star-forming gas in the two models. The stellar mass within the inner region is dominated by young stars out to $220 \mathrm{pc}$ (see Fig. 9), where the metallicities of the young stellar particles are defined by the star-forming gas. The central depletion in the passive stellar metallicity follows from the flattened profile of the passively evolved gaseous metallicity, whereas the enriched model shows an enhanced metallicity resulting from the enriched gas (see left-hand side of Fig. 10). However, the overall stellar metallicity of the remnant is for the majority of the radial range set by dynamical evolution, as is shown by the overlap of the passively evolved and enriched profiles. The $[\mathrm{O} / \mathrm{H}]$ gradient flattens from the initial $0.06 \mathrm{dex} \mathrm{kpc}^{-1}$ to $\lesssim 0.028 \mathrm{dex} \mathrm{kpc}^{-1}$ (in the linear region $r \gtrsim 1 \mathrm{kpc}$ ) still maintaining a clearly decreasing trend towards outer radii. This is in agreement with observations of metallicities in ETGs with similar stellar masses as the Antennae remnant (e.g. Tortora et al. 2010; La Barbera et al. 2012). In general observed lower mass late-type galaxies exhibit steeper abundance gradients, whereas for ETGs with $M_{*} \gtrsim 2 \times 10^{10} \mathrm{M}_{\odot}$ the gradients flatten towards larger masses (Tortora et al. 2010), in good agreement with our simulation results.

\subsection{Velocity dispersion}

An important observable that can be derived from most surveys is the velocity dispersion. Planetary nebulae and globular clusters can be observed at radii exceeding the effective radius, where gas is seldom present in substantial quantities. Here, we analyse the intrinsic dispersion profiles and revisit in Section 7 the subject by studying the projected line-of-sight velocity dispersion. In Fig. 11, we present the intrinsic radial and tangential velocity dispersions, $\sigma_{r}(r), \sigma_{\phi}(r)$, and $\sigma_{\theta}(r)$, for the stellar particles as a function of radius.

The Antennae remnant is fairly isotropic, as the different components of the velocity dispersion remain within $50 \mathrm{~km} \mathrm{~s}^{-1}$ of each other for the entire radial range. The dispersion profiles reach

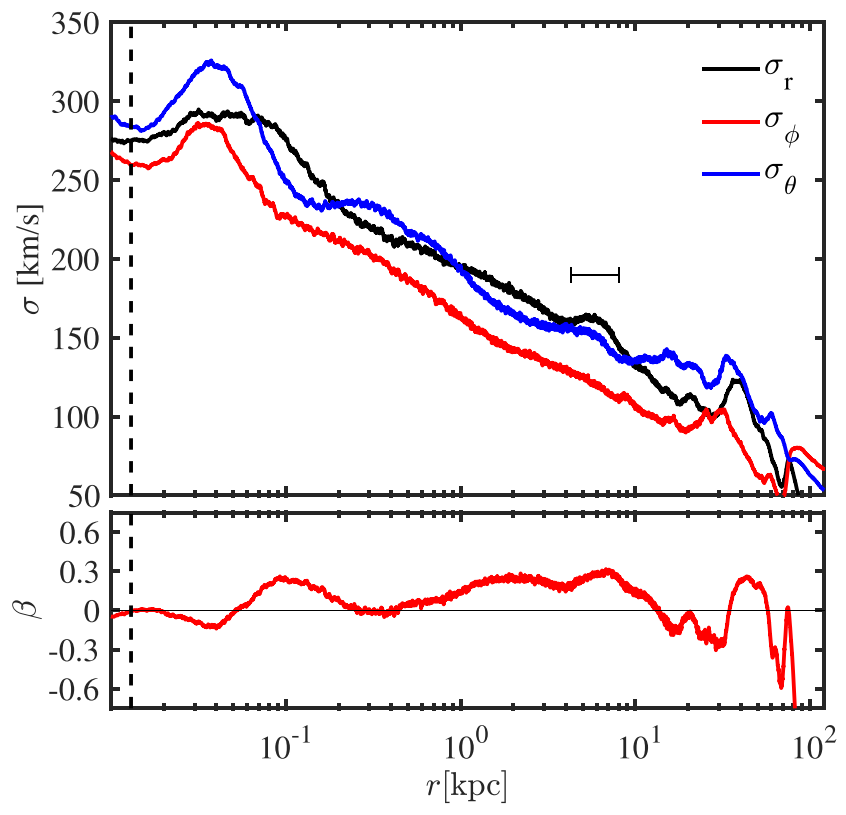

Figure 11. Top: the stellar velocity dispersion profile separated into the intrinsic radial (black) and the two tangential (red and blue) components. The bar indicates the position of the $\sigma$-bump (see the text for details). Bottom: the anisotropy profile calculated from the radial and tangential dispersion profiles as in equation (7). The dashed line in both of the panels indicates the baryonic gravitational softening length of $13 \mathrm{pc}$.

their maxima of $\sigma_{r, \max }=294 \mathrm{~km} \mathrm{~s}^{-1}, \sigma_{\phi, \max }=285 \mathrm{~km} \mathrm{~s}^{-1}$, and $\sigma_{\theta, \max }=326 \mathrm{~km} \mathrm{~s}^{-1}$ at $\sim 35 \mathrm{pc}$ and decrease fairly smoothly out to $r \sim 30 \mathrm{kpc}$. The profile is in agreement with the line-of-sight velocity dispersion of local ETGs, where the range of values is typically from 50 to $300 \mathrm{~km} \mathrm{~s}^{-1}$ measured within the effective radius (Cappellari et al. 2013). From $r \approx 40 \mathrm{kpc}$ outwards, there are multiple stellar streams, seen in projection as typical merger-induced 
shells, which are seen as locally oscillating values in the dispersion profiles.

The relation between the radial and tangential dispersion components can be more quantitatively expressed with the radial anisotropy parameter defined as

$\beta(r) \equiv 1-\frac{\sigma_{\phi}^{2}(r)+\sigma_{\theta}^{2}(r)}{2 \sigma_{r}^{2}(r)}$,

which is shown in the bottom panel of Fig. 11. The anisotropy parameter may range from $\beta \rightarrow-\infty$ for a purely rotating stellar system to $\beta=1$ for purely radial dispersion. The anisotropy parameter of the stellar component stays between $-0.3<\beta<0.31$ for most of the radial range, tending towards slightly radially biased values at intermediate radii of $0.05-13 \mathrm{kpc}$. This is in agreement with other simulation studies that found that dissipational mergers tend to produce fairly isotropic, radially biased merger remnants (Cox et al. 2006b).

In the region of $4-8 \mathrm{kpc}$, a slight $10-20 \mathrm{~km} \mathrm{~s}^{-1}$ increase in the dispersions of the $\sigma_{r}(r)$ and $\sigma_{\theta}(r)$ profiles can be seen, indicated in Fig. 11 with a horizontal bar. This type of a feature has been identified in the literature as a ' $\sigma$-bump', which differs fundamentally from the oscillating nature of the signature caused by the outer shell structures (Schauer et al. 2014). The shells can be seen as enhancements in the radial density profile, whereas the $\sigma$-bumps have not been associated with any clear structures in galaxies as is also the case in the Antennae remnant (see Fig. 9). Strong $\sigma$-bumps have also been detected only in the stellar component of simulated merger remnants.

\section{OBSERVED PHOTOMETRY OF THE MERGER REMNANT}

\subsection{Light profile}

The unobscured (purely stellar) and obscured (including dust in the ISM and the PDRs) projected surface brightness profiles of the 1-Gyr-old merger remnant are shown in Fig. 12. The profiles are calculated from the SDSS equivalent $r$-band image of the remnant as it would be seen in the sky, produced with SKIRT assuming a distance of $30 \mathrm{Mpc}$. For reference, we show the dusty profiles of both the image with the resampling of the young stellar component (PDR) and without the resampling (BC03 only). The unobscured remnant shows a cuspy profile towards the centre, caused by the concentrated star formation in the central region. The inclusion of dust obscuration suppresses the surface brightness within the entire radial range of $r<6.5 \mathrm{kpc}$, which is best seen in the dusty $\mathrm{BC} 03$ profile that excludes emission from the very youngest stellar component. The strongest obscuration of up to $3 \mathrm{mag} \mathrm{arcsec}^{-2}$ is seen within the central $2 \mathrm{kpc}$ of the dusty $\mathrm{BC} 03$ profile, which contains $\sim 3.6 \times 10^{8} \mathrm{M}_{\odot}$ of gas. We note that observed ETGs evolving towards the red sequence have been found to include significant amounts of dust and ongoing star formation (e.g. Rowlands et al. 2012). Even though the remnant is clearly an elliptical-like galaxy, at an age of $1 \mathrm{Gyr}$ it is still transitioning towards a classical red and dead ETG.

The resampling procedure introduces $1.7 \times 10^{7} \mathrm{M}_{\odot}$ of starforming regions (PDR-particles) in the inner galaxy. Including the SED of the young stellar component increases the surface brightness up to the level of the unobscured profile in the central region of the galaxy. The properly processed young stellar disc is therefore more alike the unobscured $\mathrm{BC} 03$ image within a cuspy central region,

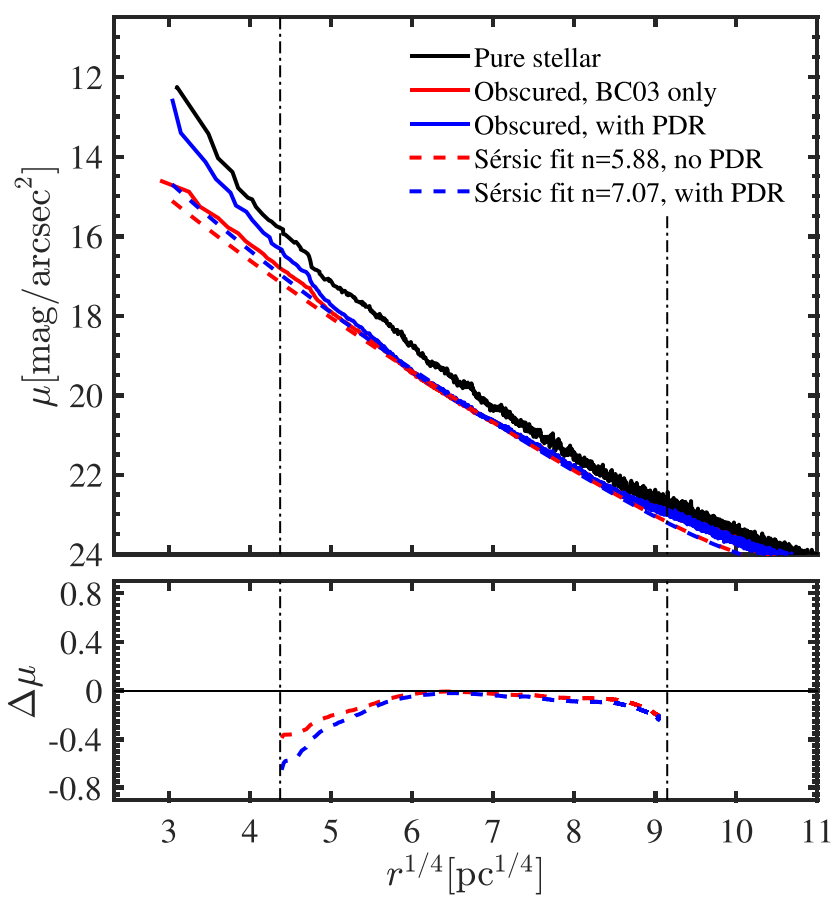

Figure 12. The unobscured (black solid line) and obscured projected surface brightness profiles in the $r$-band mock image of the 1-Gyr-old merger remnant with resampling of young stars (blue solid line) and without (red solid line), overlaid with the respective best-fitting Sérsic profiles with dashed lines. The bottom panel shows the data - fit residuals for the profile with resampling (blue) and without (red) in the fitted region. The vertical dot-dashed lines show the inner and outer fit limits given by the 2.5 arcsec mask and the median SDSS $r$-band sky value at $\sim 23 \mathrm{mag} \mathrm{arcsec}^{-2}$ (Pawlik et al., in preparation).

whereas the remainder of the light profile follows the dusty $\mathrm{BC} 03$ with reduced brightness wherever dust is present.

\subsection{Fitting the photometric profile}

Next, we use GALFIT to find the radial surface brightness profiles of the 1-Gyr-old remnant presented in Fig. 12. We fit the profiles to the $r$-band image following Krajnović et al. (2013a), who fit either a single component or a two component (disc + bulge) Sérsic profile, where the innermost 2.5 arcsec is masked. The radial surface brightness profile of an ETG can be expressed using the Sérsic profile

$I(r)=I_{\mathrm{e}} \exp \left\{b_{n}\left[\left(\frac{r}{r_{\mathrm{e}}}\right)^{1 / n}-1\right]\right\}$,

where $I_{\mathrm{e}}$ is the surface brightness at the effective radius $r_{\mathrm{e}}, n$ is the Sérsic index, and $b_{n}$ is a shape-factor dependent on $n$ as $b_{n}$ $\approx 1.9992 n-0.3271$ for $0.5<n<10$ (Capaccioli 1989). The projected surface brightness profile in mag $\operatorname{arcsec}^{-2}$ can be derived from equation (8) in the Vega system as

$\mu(r)=\mu_{\mathrm{e}}+\frac{b_{n}}{\ln 10}\left[\left(\frac{r}{r_{\mathrm{e}}}\right)^{1 / n}-1\right]$,

where $\mu_{\mathrm{e}}=-2.5 \log I_{\mathrm{e}}$ is the surface brightness at the effective radius. GALFIT provides the best-fitting values of $r_{\mathrm{e}}, \mu_{\mathrm{e}}$ and $n$ for the Sérsic profile assuming the Vega-system. For comparison to 
the ATLAS ${ }^{3 \mathrm{D}}$ data that uses asinh magnitudes, the projected surface brightness profiles are expressed in Fig. 12 in the asinh system as

$\mu(r)=-\frac{2.5}{\ln 10}\left[\operatorname{asinh}\left(\frac{f / f_{0}}{2 b}\right)+\ln b\right]$,

where $f$ is the flux, obtained for the Sérsic profile from equation (8), $f_{0}$ is the zero-point flux $3631 \mathrm{Jy}$, and $b$ is a filter-dependent constant. ${ }^{4}$ We fit both the image with and without the resampling of the young stellar component in the asinh system.

Observations have shown that ETGs typically exhibit Sérsic indices close to the de Vaucouleurs value $n \sim 4$ (Kormendy et al. 2009). If a cusp is apparent in the surface brightness profile of an ETG, an additional disc-like light profile can be fitted with a Sérsic index $n \sim 1$ (see e.g. Hopkins et al. 2009a). The central cusp is a characteristic property stemming from the central merger induced starburst. However, the innermost regions of ETGs are usually masked out which may exclude the inner disc from the fitted region. Here, following $\mathrm{ATLAS}^{3 \mathrm{D}}$ we use an equivalent mask of 2.5 arcsec in the inner galaxy and limit the fit to a median SDSS $r$-band sky value of $\sim 23 \mathrm{mag} \operatorname{arcsec}^{-2}$ at approximately $6.6 \mathrm{kpc}$.

We show the best-fitting surface brightness profiles in Fig. 12 with the residuals for both of the profiles with and without resampling of the young stars. The fits are single component Sérsic profiles, with the fitting performed between the two vertical dot-dashed lines, thus excluding the very central parts and the outer low surface brightness region. We find $r_{\mathrm{e}}=11.07$ arcsec $=1.61 \mathrm{kpc}, \mu_{\mathrm{e}}=19.81 \mathrm{mag} \operatorname{arsec}^{-2}$, and $n=7.07$ for the profile with the resampling of young stars and $r_{\mathrm{e}}=13.51 \operatorname{arcsec}=1.97 \mathrm{kpc}$, $\mu_{\mathrm{e}}=20.26 \mathrm{mag} \mathrm{arsec}^{-2}$, and $n=5.88$ for the profile without resampling. The derived Sérsic indices are within the range of typical values for local ETGs with dynamical masses $>4 \times 10^{10} \mathrm{M}_{\odot}$, where the distribution peaks at $n \approx 3-3.5$ (Kormendy et al. 2009; Krajnović et al. 2013a). However, not using the inner mask might enable the fitting of two-component profiles, thus decreasing the outer Sérsic index and making the result more similar to the earlier simulated results of Hopkins et al. (2008, 2009a,b). The effective radii are towards the lower end of the observed distribution (peak at $r_{\mathrm{e}} \approx 3-4 \mathrm{kpc}$ ), but still within the observed range (Krajnović et al. 2013a). Within the fitting region both the profiles with and without resampling of young stars are quite similar, resulting in similar Sérsic fits. In general, the fits agree with the surface brightness profiles for most of the fitted region (i.e. $\sim 1-6.5 \mathrm{kpc}$ ) and the major disagreements between the fits and the surface brightness profiles appear in the inner regions, with an increasing contribution from the central star-forming disc.

GALFIT also provides the axis ratio $b / a$ of the fitted ellipsoids at the effective radius. With a stellar mass of $\sim 10^{11} \mathrm{M}_{\odot}$, our remnant is in the intermediate mass range of ETGs and should therefore not show a high ellipticity in contrast to fast rotating lower mass elliptical galaxy. As indicated by the intrinsic shape of the stellar component in Section 5 (see also Section 8), the remnant shows only a slight elliptical shape, with $\epsilon=1-b / a=0.1-0.2$. The single component fit results in ellipticities at the effective radius of $\epsilon_{\mathrm{e}}=0.122$ and $\epsilon_{\mathrm{e}}=0.120$, compared to the intrinsic ellipticity of $\epsilon \approx 0.09$ at the same radius (see Section 5.1). The shape of the remnant is therefore consistent with being only slightly elliptical both intrinsically and also when observational and projection effects are taken into account.

\footnotetext{
${ }^{4}$ http://classic.sdss.org/dr7/algorithms/fluxcal.html
}

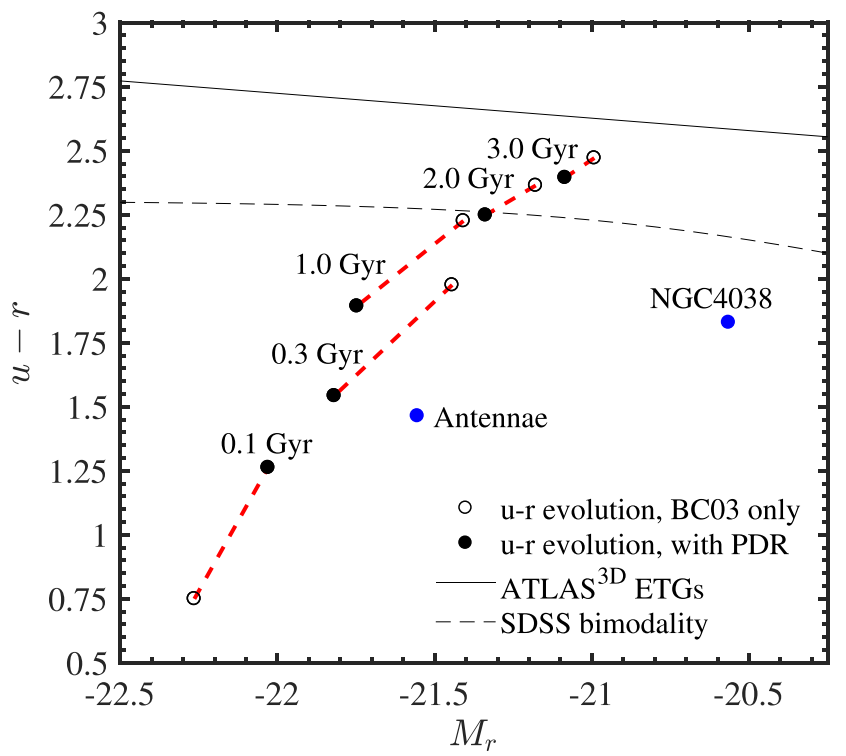

Figure 13. The evolution of the $u-r$ colour of the simulated Antennae merger as a function of the absolute $r$-band magnitude. The blue dots show the colours of the simulated NGC 4038 progenitor as well as the best match Antennae including the PDR resampling. The solid black points show the magnitude-colour evolution for the model including the resampling of the young stellar component and the open circles show the corresponding evolution for the BC03 only model without young star resampling. The colours are calculated for the remnant approximately $0.1,0.3,1,2$, and 3 Gyr after the final coalescence and the corresponding two data points are always connected by a red dashed line. The black solid line shows the bestfitting colour-magnitude relation for the local ETGs in the ATLAS ${ }^{3 \mathrm{D}}$ sample (Cappellari et al. 2011), and the dashed black line shows the division between blue and red galaxies in the SDSS survey (Baldry et al. 2004).

\subsection{Colour evolution}

The evolution of the $u-r$ colour of the remnant is shown in Fig. 13 as a function of the absolute $r$-band magnitude. The colour evolution is studied until $3 \mathrm{Gyr}$ after the coalescence. The inclusion of the resampling of the young stars provides extra blue light and shifts the remnant colours bluewards, while the $r$-band magnitude decreases mildly due to additional re-emission from dust in the volume around the star-forming regions. As a reference we show the colour of the simulated progenitor NGC 4038 (after $500 \mathrm{Myr}$ of isolated evolution) and the best match Antennae in Fig. 13, both employing the resampling of the young stellar component.

The Antennae appears $\sim 0.37$ mag bluer in the $u-r$ colour and $\sim 1$ mag brighter in the $r$-band compared to the progenitor galaxy. After the second passage $\sim 40$ Myr before the best match, the SFR has increased from $\leq 10 \mathrm{M}_{\odot} \mathrm{yr}^{-1}$ to values above $20 \mathrm{M}_{\odot} \mathrm{yr}^{-1}$. The merger appears its bluest and brightest in the $r$-band right after the final coalescence of the nuclei. As the remnant matures after coalescence, it gets dimmer and redder as the starburst subsides and the stellar population evolves predominantly passively.

Immediately after the coalescence, the residual star formation and additional dust in the starburst region can still be seen as a reddening of the colour of the 100-Myr-old remnant when comparing the MAPPINGS mincluded and BC03 only colours. After the $100 \mathrm{Myr}$ data point, the remainder of the colour evolution follows a qualitatively similar path towards the red sequence regardless of the chosen stellar library. However, for intermediate ages of $\sim 0.3-1.0 \mathrm{Gyr}$, the additional blue light in the MAPPINGS III model makes the evolution of the remnant up to 0.5 mag bluer in the $u-r$ colour when compared 
to the BC03 only colour model. The asymptotically decreasing SFR reaches its minimum of $\sim 0.8 \mathrm{M}_{\odot} \mathrm{yr}^{-1}$ after at a remnant age of $2.5 \mathrm{Gyr}$. At this age the contribution from young stars and therefore the additional MAPPINGS III flux becomes negligible. After this the spectral evolution is again governed by the increasing age of the stellar population and dominated by the BC03 SED, which drives the colour evolution of the old remnant galaxy.

As a comparison with observations, we show the best-fitting $u-r$ versus $M_{r}$ colour-magnitude relation (CMR) for ETGs in the $\operatorname{ATLAS}^{3 \mathrm{D}}$ sample with a slope $\eta=-0.097$ and zero-point $(u-r)_{0}=2.53$ in asinh magnitudes (Cappellari et al. 2011). In order to express the bimodality between the mainly blue spirals and red ETGs in the colour-magnitude plane, we also show the optimal division line between the two regimes for local SDSS galaxies with $-23.5 \leq M_{r} \leq-15.5$ (Baldry et al. 2004). Immediately after the coalescence, the merger remnant appears considerably bluer when compared to local ETGs. The $u-r$ colour increases as the remnant dims more rapidly in the $u$-band, and the $u-r$ colour approaches the observed $u-r \gtrsim 2.5 \mathrm{mag}$ value typical for ETGs with $-22 \lesssim$ $M_{r} \lesssim-18$. At an age of $\gtrsim 2.5 \mathrm{Gyr}$, the remnant crosses from the blue cloud to the red sequence, yet it still resides below the local best-fitting CMR. Cappellari et al. (2011) note that the observed colours of massive ETGs in the ATLAS ${ }^{3 \mathrm{D}}$ sample are close to the best-fitting CMR, while some of the lower mass ETGs are still only transitioning towards the relation.

\section{OBSERVED KINEMATIC STRUCTURE OF THE MERGER REMNANT}

\subsection{D line-of-sight kinematics}

Next, we compute the 2D stellar kinematics of the 1-Gyr-old remnant in the inner galaxy covering approximately 1-2 effective radii. In calculating the line-of-sight velocity distribution (LOSVD), we closely follow the procedure presented in Naab et al. (2014), which was modelled on the method used to extract LOSVD properties from the observational ATLAS ${ }^{3 \mathrm{D}}$ sample. To reduce effects from the particle mass resolution, the method replaces each stellar particle with 10 pseudo-particles with a 2D Gaussian distribution and equivalent line-of-sight velocity. The pseudo-particles are distributed with a standard deviation of $13 \mathrm{pc}$, equivalent to the gravitational softening length, which emulates also the seeing effect. These particles are then binned on a regular grid of pixels. The pixels are further grouped using the Voronoi tessellation method presented in Cappellari \& Copin (2003) to produce an irregular grid with roughly equal amount of particles in each bin. Using the maximum likelihood method, we then calculate the mean line-of-sight velocity (LOSV) $\langle V\rangle$, velocity dispersion $\sigma$, and the coefficients $h_{3}$ and $h_{4}$ representing the third and fourth moments of Gauss-Hermite series fit to the LOSVD.

Fig. 14 shows all the fitted stellar LOSVD properties of the 1Gyr-old remnant within the innermost $3.5 \mathrm{kpc}$ that encompasses approximately the area within two effective radii. The LOSVD has a spread of approximately $\langle V\rangle= \pm 60 \mathrm{~km} \mathrm{~s}^{-1}$, with a maximum velocity dispersion of $\sigma=270 \mathrm{~km} \mathrm{~s}^{-1}$ found in the centre. The rotational velocity in the central region increases by $\sim 15 \mathrm{~km} \mathrm{~s}^{-1}$ as the remnant evolves to an age of $3 \mathrm{Gyr}$. This is caused by the contribution from newly formed stars in the central rotating disc, which are added during the late evolution of the merger remnant.

Next, we compare the central region of the remnant with observed ETGs. The well-known Fundamental Plane (FP) of ETGs shows a relatively tight relation with small scatter between the velocity dispersion within the effective radius $\left\langle\sigma_{\mathrm{e}}\right\rangle$, the effective radius $r_{\mathrm{e}}$, and the surface brightness within the effective radius $\left\langle\mu_{\mathrm{e}}\right\rangle$ (Faber \& Jackson 1976; Djorgovski \& Davis 1987; Bernardi et al. 2003; Cappellari et al. 2006). For our merger remnant, the mean velocity dispersion within the effective radius $r_{\mathrm{e}} \approx 1.6 \mathrm{kpc}$ is $\left\langle\sigma_{\mathrm{e}}\right\rangle \approx$ $180 \mathrm{~km} \mathrm{~s}^{-1}$ and the mean surface brightness in the $r$-band within the effective radius is $\left\langle\mu_{\mathrm{e}}\right\rangle \approx 18.1 \mathrm{mag} \operatorname{arcsec}^{-2}$. Comparing these values to the FP fit at a corresponding $r$ band, given in Bernardi et al. (2003) for nearly 9000 ETGs in the SDSS, we find that our 1-Gyr-old merger remnant coincides almost exactly with the observed $r_{\mathrm{e}}-\left\langle\sigma_{\mathrm{e}}\right\rangle-\left\langle\mu_{\mathrm{e}}\right\rangle$ relation. However, while only 0.02 dex above the best-fitting FP, the value for the Antennae remnant is located in the small-bright-low dispersion end of the individual observations, due the bright star-forming central disc.

The distribution of $\langle V\rangle$ in Fig. 14 shows the Antennae remnant as a slightly rotating galaxy with a double maximum structure in the distribution of the mean velocity. As identified for many of the ETGs in the ATLAS ${ }^{3 \mathrm{D}}$ sample (Krajnović et al. 2011), the mean velocity reaches its maximum within the central region, then declines slightly, and reaches another maximum at a larger radius. The maxima are usually aligned, and the double maximum (2M) feature works as one of the indicators for a rotating disc in the centre of the galaxy. The central $2 \mathrm{kpc}$ with the rotating stellar disc exhibits a maximum rotation velocity of $-53 /+61 \mathrm{~km} \mathrm{~s}^{-1}$, and the other maximum of $\pm 50 \mathrm{~km} \mathrm{~s}^{-1}$ is situated at $4 \mathrm{kpc}$. The 1 -Gyr-old remnant would be classified to be a member of the most abundant class of ETGs in the ATLAS ${ }^{3 \mathrm{D}}$ sample, where 80 percent of ETGs are classified as ETGs with ordered rotation. On the other hand, in the ATLAS ${ }^{3 \mathrm{D}}$ ETG sample 14 per cent of the galaxies exhibit the double maximum (2M) feature (Krajnović et al. 2011), making this feature the second most common case after having no features at all.

An observed anticorrelation between $\langle V\rangle / \sigma$ and $h_{3}$ corresponds to ordered rotation and a disc-like axisymmetric component. This anticorrelation can be seen in the central region of the Antennae remnant in Fig. 14, given that the dispersion profile is fairly rotationally symmetric. The presence of the anticorrelation is in agreement with observations of local ETGs. For the ATLAS ${ }^{3 \mathrm{D}}$ ETGs (Krajnović et al. 2011) a $\langle V\rangle / \sigma-h_{3}$ anticorrelation is most often detected in galaxies with ordered rotation and galaxies that either have no special features, the $2 \mathrm{M}$ feature or a twist in the rotational axis of the galaxy. In addition, the $\langle V\rangle / \sigma-h_{3}$ anticorrelation is usually connected to ellipticals with relatively large angular momenta, such as ETGs formed through dissipational mergers (Naab, Jesseit \& Burkert 2006; Röttgers, Naab \& Oser 2014). Thus, the anticorrelation of $\langle V\rangle / \sigma$ and $h_{3}$ can be connected to the presence of gas and the formation of gaseous discs in the formation of ETGs, such as is the case for the Antennae remnant. We also note that the anticorrelated $\langle V\rangle / \sigma-h_{3}$ distribution remains strong for the final $2 \mathrm{Gyr}$ in the evolution of the remnant.

The $h_{4}$ coefficient illustrates a peaked $\left(h_{4}>0\right)$ or a flat topped $\left(h_{4}<0\right)$ LOSVD, where the value of $h_{4}$ roughly correlates with the anisotropy parameter $\beta$ discussed in Section 7 (Gerhard 1993; van der Marel \& Franx 1993). The distribution of $h_{4}$ encompasses predominantly positive values in Fig. 14, indicating radial anisotropy consistent with the intrinsic velocity anisotropy in Fig. 11.

\subsection{Rotation}

In order to evaluate the rotation of the remnant, we compute the $\lambda_{R}$ parameter (Emsellem et al. 2007; Naab et al. 2014) as a function of 

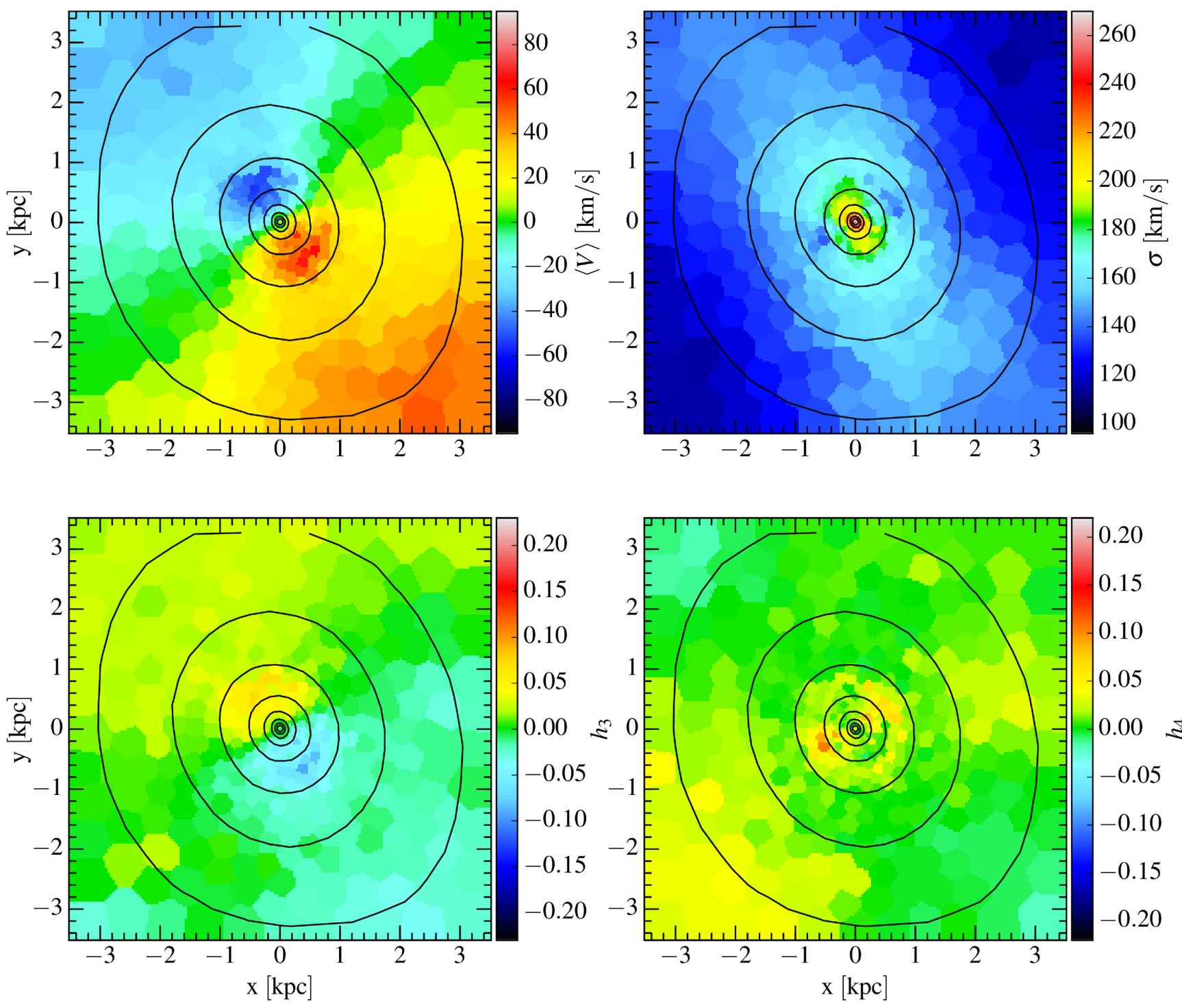

Figure 14. Mean line-of-sight velocity $\langle V\rangle$ (top left), velocity dispersion $\sigma$ (top right), and the higher order Gauss-Hermite coefficients $h_{3}$ (bottom left) and $h_{4}$ (bottom right) in the inner $3.5 \mathrm{kpc}$ (out to $\sim 2$ effective radii) of the Antennae remnant as it would be seen on the sky 1 Gyr in the future. The contours show the distribution of flux (mass) in 1 mag intervals. The range of the colour bars is adjusted to be comparable with the analysis presented in Section 8.

the projected distance of each Voronoi grid cell $i$ from the centre, defined as

$\lambda_{\mathrm{R}}=\frac{\sum_{i=1}^{N} F_{i} R_{i}\left|V_{i}\right|}{\sum_{i=1}^{N} F_{i} R_{i} \sqrt{V_{i}^{2}+\sigma_{i}^{2}}}$,

where $R_{i}$ is the projected radius, $V_{i}$ is the line-of-sight velocity, $F_{i}$ is projected flux (represented here by the mass), and $\sigma_{i}$ is the line-of-sight velocity dispersion. The sum is taken over $N$ Voronoi grid cells within the projected radius, so that the radial $\lambda_{\mathrm{R}}$ profile is cumulative.

The $\lambda_{\mathrm{R}}$ parameter can be used to divide elliptical galaxies into fast and slowly rotating galaxies with the threshold often set at $\lambda_{\mathrm{Re}}=0.1$ measured at the effective radius. A revised threshold has also been introduced by Emsellem et al. (2011), who suggest in addition to take into account also the shape of the galaxies when classifying ETGs by using a criterion $\lambda_{\operatorname{Re}}=0.31 \sqrt{\epsilon_{\mathrm{e}}}$ where $\epsilon_{\mathrm{e}}$ is the ellipticity at the effective radius. An even further refinement is provided by Cappellari (2016) who advocate a criterion with the threshold set at $\lambda_{\operatorname{Re}}=0.08+\epsilon_{\mathrm{e}} / 4$ for $\epsilon_{\mathrm{e}} \leq 0.4$, which provides a better classification for particularly round ETGs. Finally, the rotational properties of ETGs can potentially also be used to infer the formation mechanism of these galaxies (Kormendy et al. 2009), and the value of $\lambda_{\mathrm{Re}}$ usually correlates with the kinematic features of the galaxies (Cappellari et al. 2007; Krajnović et al. 2013b).

The radial distribution of stellar $\lambda_{\mathrm{R}}$ in $0.5 \mathrm{Gyr}$ intervals during the evolution of the Antennae remnant is shown in the left-hand panel of Fig. 15 . At the $r$-band effective radius of $r_{\mathrm{e}} \approx 1.6 \mathrm{kpc}$, we get a value $\lambda_{\mathrm{Re}} \approx 0.11$ for the 1-Gyr-old remnant, just above the thresholds for slow rotation. Slow rotators can be produced in binary merger simulations when one of the progenitors begins with retrograde rotation with respect to the orbital motion (Bois et al. 2011). Even though the Antennae progenitors are not counter rotating, they are both on quite inclined orbits with $i=60^{\circ}$ and thus result in a mildly rotating merger remnant. The radial rotation profiles in Fig. 15 are 

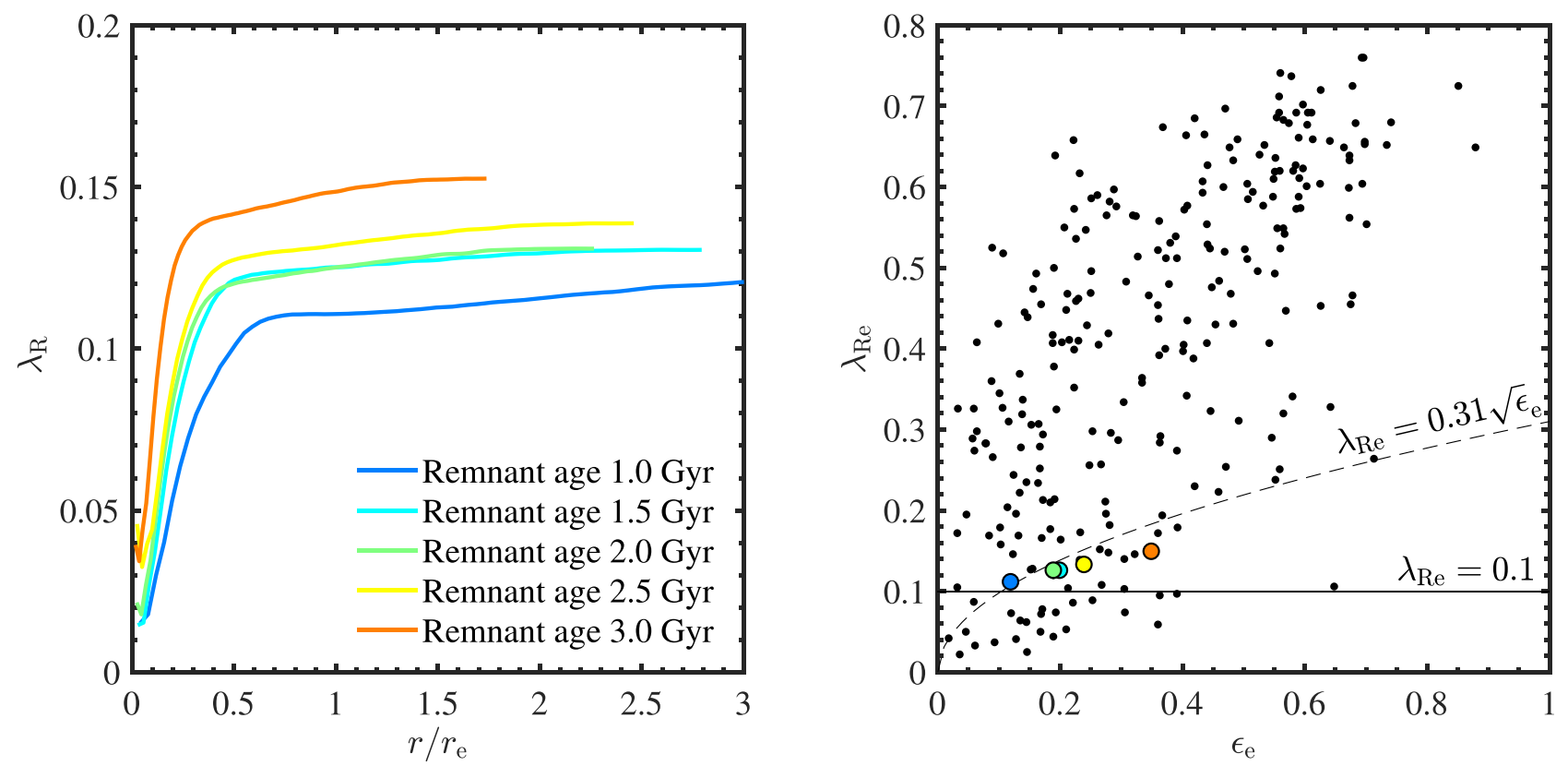

Figure 15. Left: The $\lambda_{\mathrm{R}}$-parameter as a function of radius scaled by the $r$-band effective radius. The different colours indicate the age of the remnant from 1 to $3 \mathrm{Gyr}$ and the curves span approximately $6 \mathrm{kpc}$. Right: The values of $\lambda_{\mathrm{Re}}$ during the evolution of the remnant as a function of the corresponding ellipticity, compared to the threshold values $\lambda_{\mathrm{Re}}=0.1$ (dashed) and $\lambda_{\mathrm{Re}}=0.31 \sqrt{\epsilon_{\mathrm{e}}}$ (solid). Galaxies are classified as slow rotators if they end up below the chosen threshold curve. The marker colours are the same as in the left-hand figure and the black dots show ETGs in the ATLAs ${ }^{3 \mathrm{D}}$ sample (Krajnović et al. 2011). Note the different scales on the $y$-axes.

monotonically increasing until they reach plateaus at $r>0.5 r_{\mathrm{e}}$. The radial angular momentum profiles remain fairly similar with only a slight steepening in the inner regions during the evolution of the remnant. The value of $\lambda_{R}$ evolves upwards up to a value of $\sim 0.15$ as the remnant matures to the age of $3 \mathrm{Gyr}$. This increase of angular momentum is due to the star-forming disc in the central region, as was also seen with the mean velocity distribution. If we limit our study to the evolution of the angular momentum in the stars already present in the 1-Gyr-old remnant, the $\lambda_{\mathrm{R}}$ profile does not evolve during the final $2 \mathrm{Gyr}$.

The right-hand panel of Fig. 15 shows the $\lambda_{\text {Re }}$ values for the evolving remnant as a function of the respective ellipticity, along with the threshold $\lambda_{\operatorname{Re}}$ values to aid the classification of the remnant. The curves in the right-hand panel of Fig. 15 show the difference between the traditional threshold $\lambda_{\mathrm{Re}}=0.1$ and the revised $\lambda_{\mathrm{Re}}=0.31 \sqrt{\epsilon_{\mathrm{e}}}$ threshold, and the curves demonstrate how the classification of the Antennae remnant as a fast or a slow rotator actually depends on the chosen threshold. Even though the remnant has a fairly low angular momentum for the duration of its evolution, it would still always be classified as a fast rotator using the traditional old criterion. However, the low $\lambda_{\text {Re }}$ values combined with an increasing projected ellipticity drives the remnant towards and below the revised Emsellem et al. (2011) threshold as the remnant ages. Thus in this case the remnant would be classified as a fast rotator only before it reaches approximately an age of $1 \mathrm{Gyr}$.

In addition, we also show the $\lambda_{\operatorname{Re}}-\epsilon_{\mathrm{e}}$ values for all the ETGs in the ATLAS ${ }^{3 \mathrm{D}}$ sample in the right-hand panel of Fig. 15, obtained from Emsellem et al. (2011). As the scatter in the observed $\lambda_{\mathrm{Re}}-\epsilon_{\mathrm{e}}$ values of these ETGs is rather large, correlations with additional observables have also been studied to more consistently classify ETGs. The value of $\lambda_{\mathrm{Re}}$ is commonly compared with the stellar mass. Massive ETGs $\left(M_{\text {dyn }}>10^{11.5} \mathrm{M}_{\odot}\right)$ in the ATLAS ${ }^{3 \mathrm{D}}$ sample mainly populate the low $\epsilon_{\mathrm{e}}$, low $\lambda_{\mathrm{Re}}$ part of the $\lambda_{\mathrm{Re}}-\epsilon_{\mathrm{e}}$ diagram that coincides with the slow rotator definition given in Cappellari (2016). Meanwhile, the lower mass ETGs similar to the Antennae remnant fill a much larger area in the $\lambda_{\mathrm{Re}}-\epsilon_{\mathrm{e}}$ diagram (Emsellem et al. 2011), including the region into which the Antennae remnant evolves.

\section{FINDING THE LOCAL COUNTERPARTS}

\subsection{Local ATLAS ${ }^{3 \mathrm{D}}$ counterparts}

We have shown in Sections 6 and 7 that the Antennae merger remnant will evolve into an ETG with properties in good agreement with local observed ETGs. We now search the ATLAS ${ }^{3 \mathrm{D}}$ sample for the most promising look-alikes in the local universe, as a comparison for the 1-Gyr-old Antennae merger remnant.

The ATLAS ${ }^{3 \mathrm{D}}$ galaxies are provided with $T$ types (de Vaucouleurs 1959,1963 ) and classified in the survey as either S0 if $T>-3.5$ or ellipticals if $T \leq-3.5$ (Cappellari et al. 2011). We begin by considering only galaxies that have originally been classified as ellipticals. To narrow down the search, we take the luminosities and mass-to-light ratios $(M / L)$ from Cappellari et al. (2013) obtained with the Jeans Anisotropic Modelling (JAM) ${ }^{5}$ method (Cappellari 2008) and identify ETGs with masses similar to the stellar mass of the remnant, $M_{*}=10.4 \times 10^{10} \mathrm{M}_{\odot}$. The stellar mass estimates for the ATLAS ${ }^{3 \mathrm{D}}$ galaxies are obtained from Cappellari et al. (2013) as a function of the modelled luminosity $L$ in the $r$ band as

\footnotetext{
${ }^{5} \mathrm{JAM}$ expands the widely used Jeans equations to account for anisotropic velocity distributions. The method uses observed surface brightness photometry and integral field observations to extract for example the inclinations and mass-to-light ratios of galaxies with multiple kinematic components and spatially varying anisotropies.
} 
Table 4. Observed properties of the best look-alikes of the Antennae merger remnant in the ATLAS ${ }^{3 \mathrm{D}}$ sample. Properties derived from the mock images are provided both with and without the resampling of the young stars.

\begin{tabular}{|c|c|c|c|c|c|}
\hline Property & 1-Gyr-old remnant & NGC 3226 & NGC 3379 & NGC 4494 & Most similar \\
\hline$M_{*}{ }^{a}$ & 10.42 & 9.84 & 8.22 & 9.84 & NGC 3226/NGC 4494 \\
\hline$\epsilon_{\mathrm{e}}^{b}$ & 0.12 & 0.168 & 0.13 & 0.173 & NGC 3379 \\
\hline$r_{\mathrm{e}}^{c}$ & $1.61 / 1.97$ & 7.26 & 2.48 & 3.62 & NGC 3379 \\
\hline$\mu_{\mathrm{e}}^{d}$ & $19.81 / 20.26$ & 22.95 & 20.83 & 21.03 & NGC 3379 \\
\hline$n^{e}$ & $7.07 / 5.77$ & 4.6 & 5.3 & 3.4 & NGC 3379 \\
\hline$\lambda_{\operatorname{Re}^{f}}^{f}$ & 0.11 & 0.251 & 0.157 & 0.212 & NGC 3379 \\
\hline$\langle V\rangle^{g}$ & $-53 /+61$ & $-81 /+90$ & $-90 /+93$ & $-94 /+87$ & NGC 3226 \\
\hline$\sigma$ & $100 / 268$ & $97 / 216$ & $113 / 395$ & $96 / 195$ & NGC 3226 \\
\hline Kinematic features & $2\langle V\rangle$ maxima & - & - & $2\langle V\rangle$ maxima & NGC 4494 \\
\hline
\end{tabular}

Notes. ${ }^{a}$ Total stellar mass for the simulated remnant, $M_{*} \approx L \times(M / L)$ for the $\operatorname{ATLAS}^{3 \mathrm{D}}$ galaxies (see the text for details) in units of $10^{10} \mathrm{M}_{\odot}$.

${ }^{b}$ Ellipticity at $r_{\mathrm{e}}$.

${ }^{c}$ Single component fit effective radius at $r$-band in kpc, assuming the distance estimates given in Cappellari et al. (2011).

${ }^{d}$ Single component fit surface brightness at effective radius in mag $\operatorname{arcsec}^{-2}$ at $r$-band.

${ }^{e}$ Single component fit Sérsic index at $r$ band.

${ }^{g}$ Specific angular momentum at $r$-band effective radius; velocities in $\mathrm{km} \mathrm{s}^{-1}$.

$M_{*} \approx L \times(M / L)$, assuming that the modelled $M / L$ is representative of the luminous matter within the inner galaxy where contribution from dark matter is assumed to be relatively low.

There are a few elliptical galaxies within a stellar mass range of $(10 \pm 2) \times 10^{10} \mathrm{M}_{\odot}$. The final selection of candidates is done based on the observed kinematic structure: many ellipticals with suitable masses and ellipticities have clearly different rotational or kinematic properties and we omit those galaxies from our sample. Finally, three ellipticals, NGC 3226, NGC 3379, and NGC 4494 remain. These galaxies are classified in the ATLAS ${ }^{3 \mathrm{D}}$ sample as having similar kinematic properties to our remnant with regular rotation, fairly low $\lambda_{\operatorname{Re}}$ values and either no special kinematic features as is the case with NGC 3226 and NGC 3379 or a aligned double maxima in $\langle V\rangle$ as is the case for NGC 4494 (Krajnović et al. 2011).

The characteristic values describing the kinematic structure and surface brightness profiles used in the selection of the best candidates are listed for the three galaxies in Table 4 along with the respective values for the 1-Gyr-old Antennae remnant. The final column in Table 4 indicates which of the galaxies is identified as the best look-alike in each category.

\subsection{Photometric properties}

In Fig. 16, we show the best-fitting single component surface brightness profiles for the three ellipticals obtained from Krajnović et al. (2013a), together with the profile of the 1-Gyr-old Antennae remnant (including the resampling of young stars) and its best-fitting Sérsic profile. The best-fitting parameters for the single component Sérsic profiles of the ATLAS ${ }^{3 \mathrm{D}}$ ellipticals ${ }^{6}$ are summarized in Table 4 . All the chosen candidates span a very similar range of surface brightnesses. This confirms that the photometric profile of the Antennae remnant is in good agreement with observations of the local ETG population with similar masses. Although the observed galaxies could have extra-light in the centres, the light profiles of the ATLAS ${ }^{3 \mathrm{D}}$ galaxies by applying the mask discussed in Section 6

\footnotetext{
${ }^{6}$ The one-dimensional best-fitting to the surface brightness profiles has been obtained by azimuthally averaging the light distribution in the images along the best-fitting ellipses found using the KINEMETRY code (Krajnović et al. 2006) and by minimizing the variations of the fitted intensity along the ideal ellipses at each radius.
}

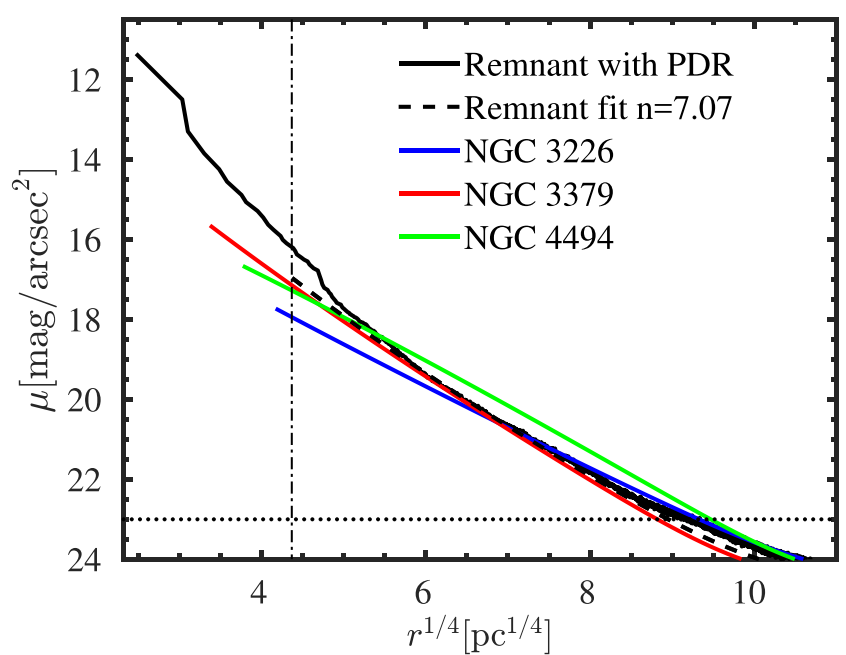

Figure 16. $r$-band surface brightness profile of the 1-Gyr-old Antennae remnant (solid black, including the resampling of the young stars) and its best-fitting Sérsic profile (dashed black), compared to the best-fitting profiles of the selected ATLAS ${ }^{3 \mathrm{D}}$ look-alikes (coloured lines). The dotted line shows the mean SDSS sky value indicating a typical outer limit for the light profile fitting of ATLAS ${ }^{3 \mathrm{D}}$ galaxies, and the dot dashed line shows the inner mask limit of 2.5 arcsec used in the fitting of light profiles of the Antennae remnant. The Sérsic profiles are only shown from the respective 2.5 arcsec mask outwards. NGC 3379 has a Sérsic profile very similar to the best-fitting profile of the Antennae remnant.

and are therefore compared to our remnant only in the fitted radial range.

The surface brightness profile of NGC 3379 shows a striking similarity with the mock profile of our remnant in Fig. 16, and it also has the smallest effective radius and largest effective brightness amongst the ETG candidates. Based on the profiles in Fig. 16, we show in Fig. 17 the $g_{-}, r$-, and $i$-band composite image of NGC 3379, obtained from the SDSS SkyServer, ${ }^{7}$ as the visual look-alike of the Antennae remnant. The observed image can be directly compared to the $g, r$, and $i$ composites of the 1- and 3-Gyr-old Antennae remnant in Fig. 17, constructed with a similar technique as used for the SDSS

\footnotetext{
${ }^{7}$ SDSS DR7 (Abazajian et al. 2009), http://skyserver.sdss.org/dr7/en/
} 

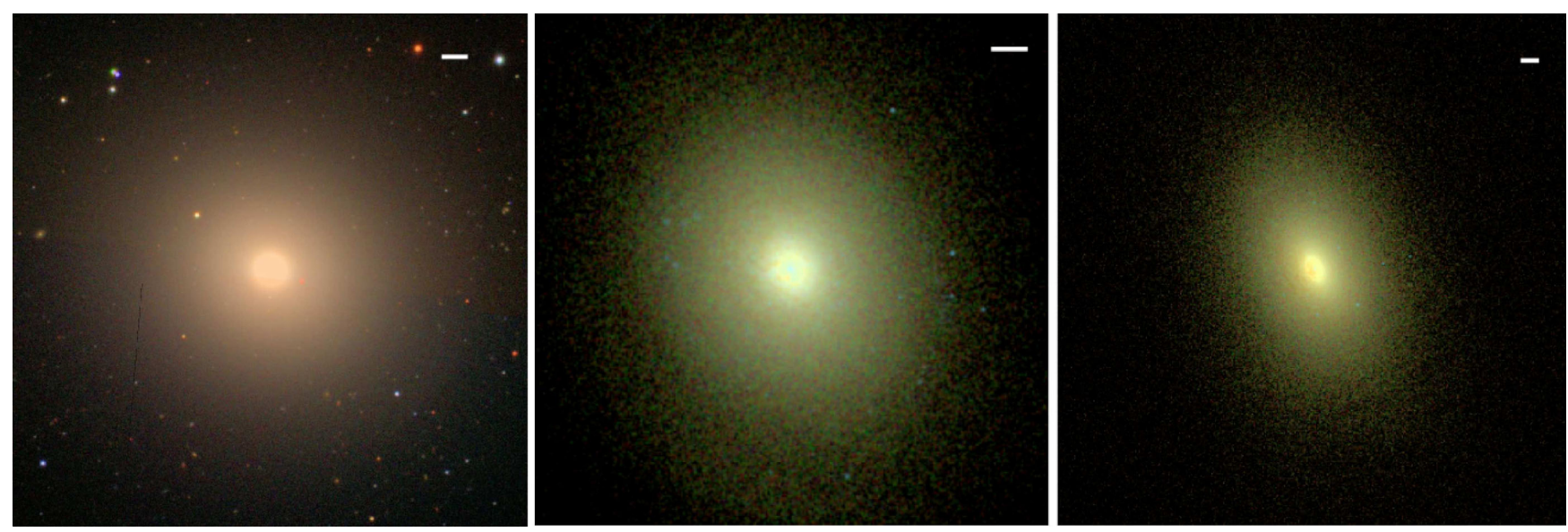

Figure 17. Left: $g, r$, and $i$ band colour-composite image of the ATLAS ${ }^{3 \mathrm{D}}$ galaxy NGC 3379 in SDSS DR7 obtained from the SDSS SkyServer. The image spans 10 times the effective radii provided in Cappellari et al. (2011). Middle and right: $g_{-}, r-$, and $i$ colour composite image of the remnant at 1 and 3 Gyr after the final coalescence of the progenitor galaxies, produced with similar photometric stretch and asinh-scaling as the ATLAs ${ }^{3 \mathrm{D}}$ image in the left-hand panel. The images are produced at the SDSS resolution of $0.396 \mathrm{arcsec}_{\mathrm{pix}}{ }^{-1}$ with SKIRT (see Section 3 ) including the resampling of the young stellar particles. The images span roughly 10 times the $r$-band effective radius of the respective galaxies and the bars show a scale of $1 \mathrm{kpc}$.

composite images, showing the same extent of 10 effective radii. The effective radius of the Antennae remnant increases by a factor of $\sim 1.5$, as the galaxy evolves, thus the $3 \mathrm{Gyr}$ image spans a larger region. The image of NGC 3379 has a better spatial resolution as the distance of NGC 3379 has been approximated to be $10.3 \mathrm{Mpc}$, $\sim 3$ times closer than our chosen distance for the Antennae.

\subsection{Kinematics}

All three candidates have slightly larger $\lambda_{\operatorname{Re}}$ values compared to the Antennae remnant, which is expected since for observed galaxies in this mass range the values of $\lambda_{\operatorname{Re}}$ span the entire range from $\lambda_{\operatorname{Re}}=0$ to $\lambda_{\mathrm{Re}} \rightarrow 1$ (see e.g. fig. 3 in Emsellem et al. 2011). Again NGC 3379 provides the most similar value $\lambda_{\mathrm{Re}}=0.157$ when compared to the $\lambda_{\operatorname{Re}}=0.12$ value of the Antennae. As the ellipticity of NGC 3379 is very similar to our simulated remnant, NGC 3379 is found very near to our remnant in the right-hand panel of Fig. 15. NGC 3379 and NGC 4494 have qualitatively similar radial $\lambda_{R}$ profiles as the Antennae remnant, based on measurements of $\lambda_{\mathrm{R}}$ at $r_{\mathrm{e}}$ and $r_{\mathrm{e}} / 2$ (Emsellem et al. 2011). The $\lambda_{\mathrm{R}}$ values are either smaller or equal at the half effective radii compared to the $\lambda_{\mathrm{R}}$ at the effective radius. NGC 3226, on the other hand, may have a centrally peaked, radially decreasing $\lambda_{\mathrm{R}}$ profile as the $\lambda_{\mathrm{R}}$ at $r_{e / 2}$ is slightly larger than at $r_{e}$.

The range of $\langle V\rangle$ for all the three observed galaxies is slightly larger than for the Antennae, while only for NGC 3379 the velocity dispersion of the central region exceeds that of the Antennae remnant. Fig. 18, which can be directly compared to the corresponding Fig. 14, shows the velocity distribution of NGC 4494, where a double $\langle V\rangle$ maximum feature can be seen within the innermost $500 \mathrm{pc}$. In addition, a slight $V / \sigma-h_{3}$ anticorrelation in the velocity distribution can be observed, similar to what is seen in the Antennae remnant. The velocity dispersion of NGC 4494, on the other hand, does not show a strong central peak, and when studying the range of LOSVD values of the Antennae remnant, NGC 3226 would be a better look-alike (see Table 4).

In conclusion, all of these observed candidates show some indisputable similarities with the simulated merger remnant of the Antennae galaxies. The surface brightness profile of NGC 3379, shown in Fig. 17, makes it a good example for the potential future visual appearance of the Antennae remnant, while NGC 3226 represents an example of what kinematic observations of the future remnant might look like. On the other hand, NGC 4494 provides an example of the commonly observed double maximum feature in the mean velocity distribution that works as an indicator for a central disc structure.

\section{CONCLUSIONS}

We have run and analysed a high-resolution hydrodynamical simulation representing the evolution of the Antennae galaxy merger (NGC 4038/4039) up to $3.2 \mathrm{Gyr}$ into the future. The use of postprocessing methods enabled a direct comparison to observations of both the Antennae system and local ETGs. Full radiative transfer modelling from ultra-violet to far-infrared wavelengths has been performed for the first time on the simulated particle data of an Antennae look-alike. Based on the spatial infrared SED, we have derived a ratio of 4.5 for the SFR in the overlap region versus the SFR in the nuclei for the simulated best-match Antennae, a value close to the observed ratio of $\sim 3-6$. In total, we obtain an SFR of $23.6 \mathrm{M}_{\odot} \mathrm{yr}^{-1}$ for the simulated Antennae, which slightly exceeds the observed value of $22.2 \mathrm{M}_{\odot} \mathrm{yr}^{-1}$. The match between the simulations and the observations could primarily be improved by tuning the initial conditions of the NGC 4039 progenitor in order to produce a lower star formation level at the time of the best match.

The refined disc galaxy initial conditions with initial metallicity and age distributions motivated by observations of the Milky Way have enabled the first simulation of the spatial stellar metallicity distribution in the Antennae system. Our metallicity values, which mostly range from solar to slightly supersolar, are in good agreement with the metallicities of observed young stellar clusters located in off-nuclear sites (Bastian et al. 2009; Lardo et al. 2015). However, the simulated metallicity in the nuclei of the still distinct galactic discs were at most $0.7 \mathrm{Z}_{\odot}$ higher than observed. A more observationally motivated analysis would therefore be warranted, which would use metallicity estimates based on spectral line indices and an observationally equivalent selection of stellar clusters based on cluster ages. 

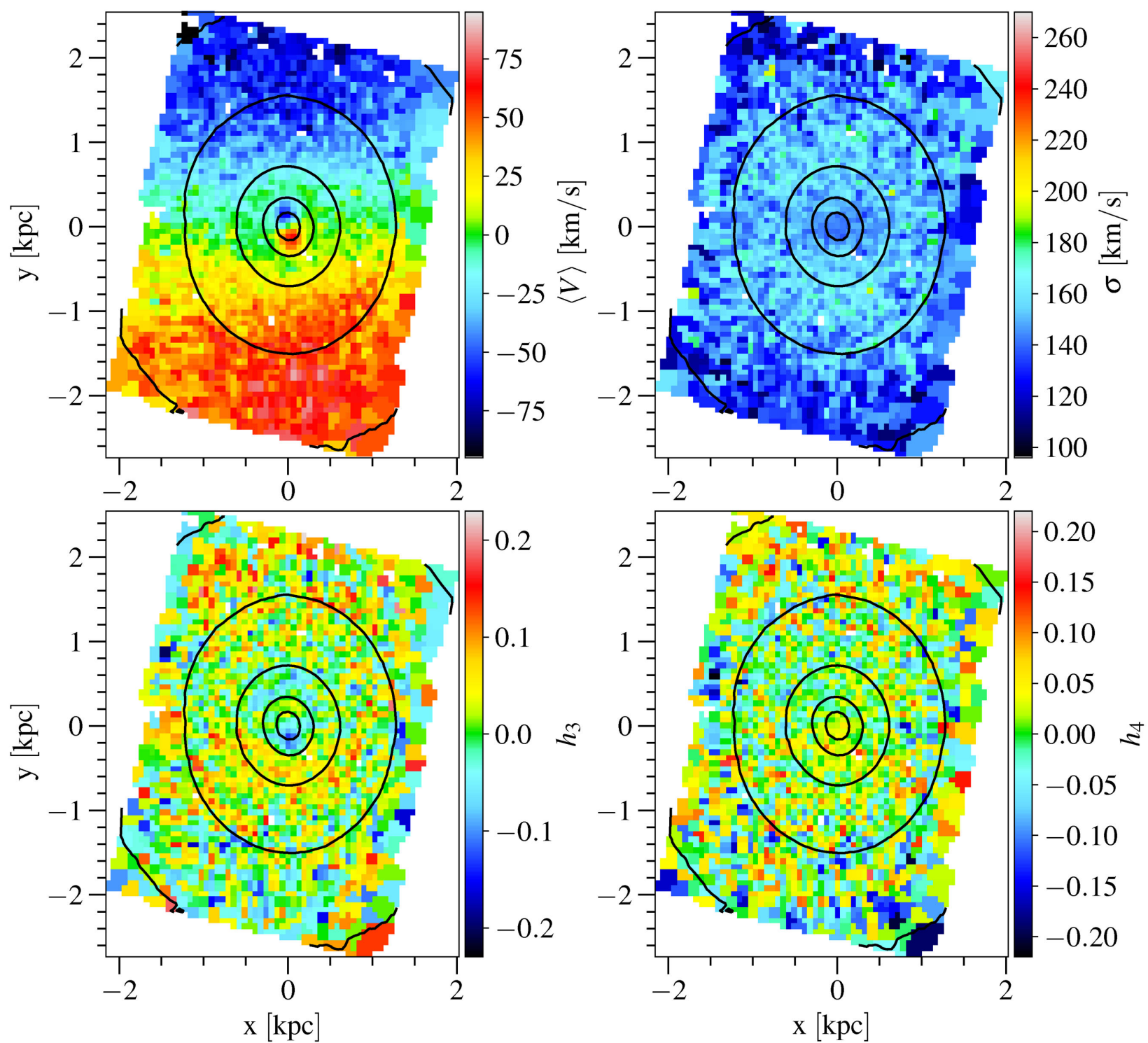

Figure 18. Same as in Fig. 14 for the ATLAS ${ }^{3 \mathrm{D}}$ galaxy NGC 4494 (Cappellari et al. 2011; Krajnović et al. 2011) showing the mean line-of-sight velocity $\langle V\rangle$ (top left), velocity dispersion $\sigma$ (top right), and the higher order Gauss-Hermite coefficients $h_{3}$ (bottom left) and $h_{4}$ (bottom right). The contours show the distribution of flux in 1 mag intervals and the LOSVD ranges are the same as in Fig. 14.

The photometric and kinematic properties of the 1-Gyr-old Antennae merger remnant has been scrutinized in detail, with the results compared to observational surveys of ETGs, in particular the ATLAS $^{3 \mathrm{D}}$ survey. The analysis of the shape of the remnant reveals a fairly spherical, isotropic galaxy with intrinsic axis ratios predominantly in the range of $0.8-0.9$ for the entire radial range. The observed shape, including dust obscuration and projection effects, calculated by fitting a Sérsic profile to the surface brightness profile of the post-processed SDSS equivalent $r$-band image, results in an ellipticity at $r_{\mathrm{e}}$ of $\epsilon_{\mathrm{e}}=0.122$. This is only slightly larger than the respective intrinsic value of $\epsilon_{\mathrm{e}} \approx 0.09$. The best-fitting light profile has a Sérsic index of $n=7.07$, which is towards the upper end of typical values for local ETGs. The $r$-band effective radius $r_{\mathrm{e}}=1.6 \mathrm{kpc}$ of the 1-Gyr-old remnant is relatively small and this is primarily caused by the nuclear extra-light in the central disc. As a consequence, the central properties within $r_{\mathrm{e}}$ place the 1-Gyr-old remnant on the observed Fundamental Plane of ETGs, but towards the bright and compact end of the observed range. During the evo- lution of the merger remnant, the effective radius increases as the central light fades after most of the star formation in the central gaseous disc is extinguished.

The negative intrinsic radial metallicity abundance gradient of the stellar particles flattens from the initial $0.06 \mathrm{dex} \mathrm{kpc}^{-1}$ value to $0.028 \mathrm{dex} \mathrm{kpc}^{-1}$ in the 1-Gyr-old remnant, while the gaseous oxygen abundance shows a more centrally peaked distribution with a flatter gradient in the outer regions. The resulting radially decreasing abundances agree with the overall trends in metallicities of observed ETGs. The central oxygen abundances of both stars and gas are enhanced with respect to the initial conditions due to stellar enrichment and star formation from enriched gas.

After coalescence, the young remnant shows observational properties reminiscent of later-type galaxies, as the apparent $u-r$ colour is initially quite blue. The remnant shows an asymptotically decreasing amount of star formation in its central region after the merger induced starburst has faded. As a result the young stars initially prevent the remnant from crossing in the $u-r$ versus $M_{r}$ 
colour-magnitude diagram from the blue cloud to the ETG dominated red sequence. Only after $2.5 \mathrm{Gyr}$ of isolated evolution can the remnant be identified as a red ETG. In a similar manner, some local ETGs with colours bluer than expected in the ATLAS ${ }^{3 \mathrm{D}}$ sample have been identified as possibly transitioning objects between the blue cloud and the red sequence.

The spatial kinematic analysis of the stellar particles of the Antennae remnant shows a LOSVD with clearly ordered rotation. The double maximum feature in the $\langle V\rangle$ distribution of the remnant is a common feature, also seen in local ATLAS ${ }^{3 \mathrm{D}}$ ETGs. The central star-forming disc is manifested in the observational features as the double maxima in $\langle V\rangle$ and a clearly anticorrelated $\langle V\rangle / \sigma-h_{3}$ distribution, which are a natural consequence of a gas-rich major merger.

The fairly slow rotational velocity in the evolving remnant is a consequence of the orbital configuration of the Antennae galaxies: the progenitors merge with highly inclined disc orientations, with $i=60^{\circ}$ with respect to the direction of the orbital angular momentum. This results in a fairly low angular momentum in the final merger remnant. The specific angular momentum $\lambda_{\operatorname{Re}}$ at the effective radius is used to quantify the rotation, and we obtain values increasing from $\lambda_{\mathrm{Re}}=0.11$ to 0.14 during the final $2 \mathrm{Gyr}$ of evolution. The revised threshold of $\lambda_{\mathrm{Re}}=0.31 \sqrt{\epsilon_{\mathrm{e}}}$ for slow versus fast rotation would classify the remnant as a slow rotator for remnant ages $>1 \mathrm{Gyr}$, whereas the traditional threshold $\lambda_{\mathrm{Re}}=0.1$ would classify the remnant as a fast rotator throughout its evolution.

Finally, we identified three local ETGs in the ATLAS ${ }^{3 \mathrm{D}}$ sample with similar masses and photometric and kinematic properties reminiscent of the values derived for the 1-Gyr-old Antennae merger remnant. A visual counterpart with a similar surface brightness profile is provided by NGC 3379, whereas NGC 3226 has LOSVD properties in a similar range, and NGC 4494 shows the same kind of double maximum feature as was found for the Antennae remnant. Based on the kinematic features mainly dominated by the presence of the nuclear disc the Antennae remnant would be classified in the second most common class of local ATLAS ${ }^{3 \mathrm{D}}$ ETGs. This class includes 14 per cent of the ATLAS ${ }^{3 \mathrm{D}}$ ETG sample, exhibiting ordered rotation and a special, yet not entirely decoupled, kinematic feature. Overall, we conclude that the famous Antennae merger will evolve into an ETG with properties very similar to the observed properties of the general local ETG population.

\section{ACKNOWLEDGEMENTS}

The authors would like to thank Pauli Pihajoki and Till Sawala for helpful discussions and comments on the manuscript, and Milena Pawlik for help in producing the SDSS equivalent images of the simulated outputs. The numerical simulations were performed on facilities hosted by the CSC-IT Center for Science Ltd. in Espoo, Finland. N.L., P.H.J, and A.R. acknowledge support from the Max Planck Institute for Astrophysics Garching Visitor Programme. N.L. is supported by the Jenny ja Antti Wihurin Rahasto and the Doctoral Programme in Particle Physics and Universe Sciences at the University of Helsinki. N.L., P.H.J., and A.R. acknowledge the support of the Academy of Finland grant 274931. A.R. is funded by the Doctoral Programme in Particle Physics and Universe Sciences at the University of Helsinki.

\section{REFERENCES}

Aarseth S. J., Fall S. M., 1980, ApJ, 236, 43

Abazajian K. N. et al., 2009, ApJS, 182, 543

Adelman S. J., Robinson R. D., Wahlgren G. M., 1993, PASP, 105, 327
Asplund M., Grevesse N., Sauval A. J., Scott P., 2009, ARA\&A, 47, 481

Aumer M., White S. D. M., Naab T., Scannapieco C., 2013, MNRAS, 434, 3142

Baldry I. K., Glazebrook K., Brinkmann J., Ivezić Ž., Lupton R. H., Nichol R. C., Szalay A. S., 2004, ApJ, 600, 681

Barnes J. E., 1988, ApJ, 331, 699

Barnes J. E., Hernquist L. E., 1991, ApJ, 370, L65

Barnes J. E., Hernquist L., 1992, ARA\&A, 30, 705

Barnes J. E., Hernquist L., 1996, ApJ, 471, 115

Bastian N., Trancho G., Konstantopoulos I. S., Miller B. W., 2009, ApJ, 701,607

Bender R., Saglia R. P., Gerhard O. E., 1994, MNRAS, 269, 785

Bernardi M. et al., 2003, AJ, 125, 1866

Bessell M. S., Castelli F., Plez B., 1998, A\&A, 333, 231

Bigiel F., Leroy A. K., Blitz L., Bolatto A. D., da Cunha E., Rosolowsky E., Sandstrom K., Usero A., 2015, ApJ, 815, 103

Blondin J. M., Wright E. B., Borkowski K. J., Reynolds S. P., 1998, ApJ, 500,342

Bois M. et al., 2011, MNRAS, 416, 1654

Bolton A. S., Burles S., Koopmans L. V. E., Treu T., Moustakas L. A., 2006, ApJ, 638, 703

Bolton A. S., Burles S., Koopmans L. V. E., Treu T., Gavazzi R., Moustakas L. A., Wayth R., Schlegel D. J., 2008, ApJ, 682, 964

Bournaud F., Jog C. J., Combes F., 2005, A\&A, 437, 69

Brandl B. R. et al., 2009, ApJ, 699, 1982

Bruzual G., Charlot S., 2003, MNRAS, 344, 1000 (BC03)

Bryant J. J. et al., 2015, MNRAS, 447, 2857

Bullock J. S., Dekel A., Kolatt T. S., Kravtsov A. V., Klypin A. A., Porciani C., Primack J. R., 2001, ApJ, 555, 240

Camps P., Baes M., 2015, Astron. Comput., 9, 20

Camps P., Trayford J. W., Baes M., Theuns T., Schaller M., Schaye J., 2016, MNRAS, 462, 1057

Capaccioli M., 1989, in Corwin H. G., Jr., Bottinelli L., eds, World of Galaxies (Le Monde des Galaxies). Springer-Verlag, New York, p. 208 Cappellari M., 2008, MNRAS, 390, 71

Cappellari M., 2016, ARA\&A, 54, 597

Cappellari M., Copin Y., 2003, MNRAS, 342, 345

Cappellari M. et al., 2006, MNRAS, 366, 1126

Cappellari M. et al., 2007, MNRAS, 379, 418

Cappellari M. et al., 2011, MNRAS, 413, 813

Cappellari M. et al., 2013, MNRAS, 432, 1709

Casagrande L., Flynn C., Portinari L., Girardi L., Jimenez R., 2007, MNRAS, 382, 1516

Chabrier G., 2003, PASP, 115, 763

Cioffi D. F., McKee C. F., Bertschinger E., 1988, ApJ, 334, 252

Cox T. J., Jonsson P., Primack J. R., Somerville R. S., 2006a, MNRAS, 373 , 1013

Cox T. J., Dutta S. N., Di Matteo T., Hernquist L., Hopkins P. F., Robertson B., Springel V., 2006b, ApJ, 650, 791

Cullen L., Dehnen W., 2010, MNRAS, 408, 669

de Vaucouleurs G., 1959, Handbuch der Physik, 53, 275

de Vaucouleurs G., 1963, ApJS, 8, 31

de Vaucouleurs A., Longo G., 1988, Catalogue of visual and infrared photometry of galaxies from 0.5 micrometer to 10 micrometer (1961-1985)

de Vaucouleurs G., de Vaucouleurs A., Corwin Jr. H. G., Buta R. J., Paturel G., Fouqué P., 1991, Third Reference Catalogue of Bright Galaxies. Volume I: Explanations and references. Volume II: Data for galaxies between $0^{h}$ and $12^{h}$. Volume III: Data for galaxies between $12^{h}$ and $24^{h}$.

Dasyra K. M. et al., 2006a, ApJ, 638, 745

Dasyra K. M. et al., 2006b, ApJ, 651, 835

Davies R. L., Efstathiou G., Fall S. M., Illingworth G., Schechter P. L., 1983, ApJ, 266, 41

Dehnen W., Aly H., 2012, MNRAS, 425, 1068

Djorgovski S., Davis M., 1987, ApJ, 313, 59

Draine B. T., Lee H. M., 1984, ApJ, 285, 89

Draine B. T., Li A., 2001, ApJ, 551, 807

Dwek E., 1998, ApJ, 501, 643

Edmunds M. G., Pagel B. E. J., 1984, MNRAS, 211, 507 
Eggen O. J., Lynden-Bell D., Sandage A. R., 1962, ApJ, 136, 748

Eisenreich M., Naab T., Choi E., Ostriker J. P., Emsellem E., 2017, MNRAS, 468,751

Ellison S. L., Patton D. R., Simard L., McConnachie A. W., 2008, AJ, 135, 1877

Emsellem E. et al., 2007, MNRAS, 379, 401

Emsellem E. et al., 2011, MNRAS, 414, 888

Fabbiano G., Zezas A., Murray S. S., 2001, ApJ, 554, 1035

Faber S. M., Jackson R. E., 1976, ApJ, 204, 668

Faber S. M. et al., 1997, AJ, 114, 1771

Feldmann R., Carollo C. M., Mayer L., 2011, ApJ, 736, 88

Fiorentino G., Musella I., Marconi M., 2013, MNRAS, 434, 2866

Gavazzi R., Treu T., Rhodes J. D., Koopmans L. V. E., Bolton A. S., Burles S., Massey R. J., Moustakas L. A., 2007, ApJ, 667, 176

Genzel R. et al., 1998, ApJ, 498, 579

Gerhard O. E., 1993, MNRAS, 265, 213

Groves B., Dopita M. A., Sutherland R. S., Kewley L. J., Fischera J., Leitherer C., Brandl B., van Breugel W., 2008, ApJS, 176, 438

Guidi G., Scannapieco C., Walcher C. J., 2015, MNRAS, 454, 2381

Haardt F., Madau P., 2001, in Neumann D. M., Tran J. T. V., eds, Clusters of Galaxies and the High Redshift Universe Observed in X-rays. CEA, Saclay, p. 64

Hayward C. C., Torrey P., Springel V., Hernquist L., Vogelsberger M., 2014, MNRAS, 442, 1992

Heithausen A., Bensch F., Stutzki J., Falgarone E., Panis J. F., 1998, A\&A, 331, L65

Hernquist L., 1990, ApJ, 356, 359

Hernquist L., Katz N., 1989, ApJS, 70, 419

Herrera C. N., Boulanger F., Nesvadba N. P. H., Falgarone E., 2012, A\&A, 538, L9

Hibbard J. E., van der Hulst J. M., Barnes J. E., Rich R. M., 2001, AJ, 122, 2969

Hibbard J. E. et al., 2005, ApJ, 619, L87

Hoffman L., Cox T. J., Dutta S., Hernquist L., 2010, ApJ, 723, 818

Hopkins P. F., 2013, MNRAS, 428, 2840

Hopkins P. F., Hernquist L., Cox T. J., Dutta S. N., Rothberg B., 2008, ApJ, 679,156

Hopkins P. F., Cox T. J., Dutta S. N., Hernquist L., Kormendy J., Lauer T. R., 2009a, ApJS, 181, 135

Hopkins P. F., Lauer T. R., Cox T. J., Hernquist L., Kormendy J., 2009b, ApJS, 181, 486

Hopkins P. F., Cox T. J., Hernquist L., Narayanan D., Hayward C. C., Murray N., 2013, MNRAS, 430, 1901

Hu C.-Y., Naab T., Walch S., Moster B. P., Oser L., 2014, MNRAS, 443, 1173

Issa M. R., MacLaren I., Wolfendale A. W., 1990, A\&A, 236, 237

Iwamoto K., Brachwitz F., Nomoto K., Kishimoto N., Umeda H., Hix W. R., Thielemann F.-K., 1999, ApJS, 125, 439

Jesseit R., Cappellari M., Naab T., Emsellem E., Burkert A., 2009, MNRAS, 397, 1202

Johansson P. H., Naab T., Burkert A., 2009a, ApJ, 690, 802

Johansson P. H., Burkert A., Naab T., 2009b, ApJ, 707, L184

Johansson P. H., Naab T., Ostriker J. P., 2012, ApJ, 754, 115

Jonsson P., Groves B. A., Cox T. J., 2010, MNRAS, 403, 17

Karakas A. I., 2010, MNRAS, 403, 1413

Karl S. J., Naab T., Johansson P. H., Theis C., Boily C. M., 2008, Astronomische Nachrichten, 329, 1042

Karl S. J., Naab T., Johansson P. H., Kotarba H., Boily C. M., Renaud F., Theis C., 2010, ApJ, 715, L88

Karl S. J., Fall S. M., Naab T., 2011, ApJ, 734, 11

Karl S. J., Lunttila T., Naab T., Johansson P. H., Klaas U., Juvela M., 2013, MNRAS, 434, 696

Kennicutt R. C., Jr, 1998, ApJ, 498, 541

Kewley L. J., Ellison S. L., 2008, ApJ, 681, 1183

Kewley L. J., Rupke D., Zahid H. J., Geller M. J., Barton E. J., 2010, ApJ, 721, L48

Kilian-Montenbruck J., Gehren T., Nissen P. E., 1994, A\&A, 291, 757

Klaas U., Nielbock M., Haas M., Krause O., Schreiber J., 2010, A\&A, 518,
L44

Klypin A., Zhao H., Somerville R. S., 2002, ApJ, 573, 597

Koopmans L. V. E., Treu T., Bolton A. S., Burles S., Moustakas L. A., 2006, ApJ, 649, 599

Koopmans L. V. E. et al., 2009, ApJ, 703, L51

Kormendy J., 1977, ApJ, 218, 333

Kormendy J., Bender R., 1996, ApJ, 464, L119

Kormendy J., Bender R., 2012, ApJS, 198, 2

Kormendy J., Fisher D. B., Cornell M. E., Bender R., 2009, ApJS, 182, 216

Kotarba H., Karl S. J., Naab T., Johansson P. H., Dolag K., Lesch H., Stasyszyn F. A., 2010, ApJ, 716, 1438

Krajnović D., Cappellari M., de Zeeuw P. T., Copin Y., 2006, MNRAS, 366, 787

Krajnović D. et al., 2011, MNRAS, 414, 2923

Krajnović D. et al., 2013a, MNRAS, 432, 1768

Krajnović D. et al., 2013b, MNRAS, 433, 2812

Kramer C., Stutzki J., Rohrig R., Corneliussen U., 1998, A\&A, 329, 249

La Barbera F., Ferreras I., de Carvalho R. R., Bruzual G., Charlot S., Pasquali A., Merlin E., 2012, MNRAS, 426, 2300

Laor A., Draine B. T., 1993, ApJ, 402, 441

Lardo C., Davies B., Kudritzki R.-P., Gazak J. Z., Evans C. J., Patrick L. R., Bergemann M., Plez B., 2015, ApJ, 812, 160

Li A., Draine B. T., 2001, ApJ, 554, 778

Lupton R. H., Gunn J. E., Szalay A. S., 1999, AJ, 118, 1406

McKee C. F., Ostriker J. P., 1977, ApJ, 218, 148

McMillan P. J., 2011, MNRAS, 414, 2446

Maoz D., Mannucci F., Li W., Filippenko A. V., Della Valle M., Panagia N., 2011, MNRAS, 412, 1508

Mihos J. C., Hernquist L., 1996, ApJ, 464, 641

Mihos J. C., Bothun G. D., Richstone D. O., 1993, ApJ, 418, 82

Mirabel I. F. et al., 1998, A\&A, 333, L1

Montuori M., Di Matteo P., Lehnert M. D., Combes F., Semelin B., 2010, A\&A, 518, A56

Morris J. P., Monaghan J. J., 1997, Journal of Computational Physics, 136, 41

Morselli L., Popesso P., Erfanianfar G., Concas A., 2017, A\&A, 597, A97

Naab T., Burkert A., 2003, ApJ, 597, 893

Naab T., Ostriker J. P., 2009, ApJ, 690, 1452

Naab T., Ostriker J. P., 2017, ARA\&A, 55, 59

Naab T., Jesseit R., Burkert A., 2006, MNRAS, 372, 839

Naab T., Johansson P. H., Ostriker J. P., 2009, ApJ, 699, L178

Naab T. et al., 2014, MNRAS, 444, 3357

Negroponte J., White S. D. M., 1983, MNRAS, 205, 1009

Nelemans G., Voss R., Roelofs G., Bassa C., 2008, MNRAS, 388, 487

Núñez A., Ostriker J. P., Naab T., Oser L., Hu C.-Y., Choi E., 2017, ApJ, 836, 204

Oser L., Ostriker J. P., Naab T., Johansson P. H., Burkert A., 2010, ApJ, 725, 2312

Peng C. Y., Ho L. C., Impey C. D., Rix H.-W., 2010, AJ, 139, 2097

Perez M. J., Tissera P. B., Scannapieco C., Lambas D. G., de Rossi M. E., 2006, A\&A, 459, 361

Perez J., Michel-Dansac L., Tissera P. B., 2011, MNRAS, 417, 580

Price D. J., 2008, J. Comput. Phys., 227, 10040

Remus R.-S., Burkert A., Dolag K., Johansson P. H., Naab T., Oser L., Thomas J., 2013, ApJ, 766, 71

Remus R.-S., Dolag K., Naab T., Burkert A., Hirschmann M., Hoffmann T. L., Johansson P. H., 2017, MNRAS, 464, 3742

Renaud F., Bournaud F., Duc P.-A., 2015, MNRAS, 446, 2038

Ribas I., Jordi C., Torra J., Giménez Á., 2000, MNRAS, 313, 99

Riess A. G. et al., 2011, ApJ, 730, 119

Röttgers B., Naab T., Oser L., 2014, MNRAS, 445, 1065

Rowlands K. et al., 2012, MNRAS, 419, 2545

Rupke D. S. N., Veilleux S., Baker A. J., 2008, ApJ, 674, 172

Rupke D. S. N., Kewley L. J., Barnes J. E., 2010, ApJ, 710, L156

Saftly W., Camps P., Baes M., Gordon K. D., Vandewoude S., Rahimi A., Stalevski M., 2013, A\&A, 554, A10

Saftly W., Baes M., Camps P., 2014, A\&A, 561, A77

Saitoh T. R., Makino J., 2013, ApJ, 768, 44 
Salim S. et al., 2007, ApJS, 173, 267

Sánchez S. F. et al., 2012, A\&A, 538, A8

Sánchez S. F. et al., 2014, A\&A, 563, A49

Saviane I., Hibbard J. E., Rich R. M., 2004, AJ, 127, 660

Saviane I., Momany Y., da Costa G. S., Rich R. M., Hibbard J. E., 2008, ApJ, 678, 179

Scannapieco C., Tissera P. B., White S. D. M., Springel V., 2005, MNRAS, 364,552

Scannapieco C., Tissera P. B., White S. D. M., Springel V., 2006, MNRAS, 371,1125

Schauer A. T. P., Remus R.-S., Burkert A., Johansson P. H., 2014, ApJ, 783, L32

Schiminovich D. et al., 2007, ApJS, 173, 315

Schweizer F. et al., 2008, AJ, 136, 1482

Sedov L. I., 1959, Similarity and Dimensional Methods in Mechanics. Academic Press, New York

Shen S., Wadsley J., Stinson G., 2010, MNRAS, 407, 1581

Springel V., 2005, MNRAS, 364, 1105

Springel V., Di Matteo T., Hernquist L., 2005, MNRAS, 361, 776

Stanford S. A., Sargent A. I., Sanders D. B., Scoville N. Z., 1990, ApJ, 349, 492

Stutzki J., Bensch F., Heithausen A., Ossenkopf V., Zielinsky M., 1998, A\&A, 336, 697

Taylor G., 1950, Proc. R. Soc. A, 201, 159

Teyssier R., Chapon D., Bournaud F., 2010, ApJ, 720, L149

Thomas D., Maraston C., Bender R., Mendes de Oliveira C., 2005, ApJ, 621,673

Toomre A., 1977, in Tinsley B. M., Larson D. Campbell R. B. G., eds, Evolution of Galaxies and Stellar Populations. Yale University Observatory, New Haven, p. 401

Toomre A., Toomre J., 1972, ApJ, 178, 623
Torrey P., Cox T. J., Kewley L., Hernquist L., 2012, ApJ, 746, 108

Tortora C., Napolitano N. R., Cardone V. F., Capaccioli M., Jetzer P., Molinaro R., 2010, MNRAS, 407, 144

Trayford J. W. et al., 2017, MNRAS, 470, 771

Tremonti C. A. et al., 2004, ApJ, 613, 898

Tully R. B. et al., 2013, AJ, 146, 86

Ueda J. et al., 2012, ApJ, 745, 65

van der Marel R. P., Franx M., 1993, ApJ, 407, 525

Väisänen P., Reunanen J., Kotilainen J., Mattila S., Johansson P. H., Ramphul R., Romero-Cañizales C., Kuncarayakti H., 2017, MNRAS, 471, 2059

Wang Z. et al., 2004, ApJS, 154, 193

Wellons S. et al., 2015, MNRAS, 449, 361

White S. D. M., 1978, MNRAS, 184, 185

White S. D. M., Rees M. J., 1978, MNRAS, 183, 341

Whitmore B. C., Zhang Q., Leitherer C., Fall S. M., Schweizer F., Miller B. W., 1999, AJ, 118, 1551

Whitmore B. C. et al., 2014, ApJ, 795, 156

Wiersma R. P. C., Schaye J., Smith B. D., 2009, MNRAS, 393, 99

Wild V. et al., 2014, A\&A, 567, A132

Woosley S. E., Weaver T. A., 1995, ApJS, 101, 181

Zaritsky D., Kennicutt R. C., Jr, Huchra J. P., 1994, ApJ, 420, 87

Zemp M., Gnedin O. Y., Gnedin N. Y., Kravtsov A. V., 2011, ApJS, 197, 30

Zezas A., Fabbiano G., Baldi A., Schweizer F., King A. R., Ponman T. J.,

Rots A. H., 2006, ApJS, 166, 211

Zhang Q., Fall S. M., 1999, ApJ, 527, L81

Zhang Q., Fall S. M., Whitmore B. C., 2001, ApJ, 561, 727

Zubko V., Dwek E., Arendt R. G., 2004, ApJS, 152, 211

This paper has been typeset from a $\mathrm{T}_{\mathrm{E}} \mathrm{X} / \mathrm{L} \mathrm{T} \mathrm{E}$ file prepared by the author. 